\title{
A 5D view of the $\alpha$ Per, Pleiades, and Praesepe clusters ${ }^{\star}$
}

\author{
N. Lodieu ${ }^{1,2}$, A. Pérez-Garrido ${ }^{3}$, R. L. Smart ${ }^{4}$, and R. Silvotti ${ }^{4}$ \\ 1 Instituto de Astrofísica de Canarias (IAC), Calle Vía Láctea s/n, 38200 La Laguna, Tenerife, Spain \\ e-mail: nlodieu@iac.es \\ 2 Departamento de Astrofísica, Universidad de La Laguna (ULL), 38206 La Laguna, Tenerife, Spain \\ 3 Dpto. Física Aplicada, Universidad Politécnica de Cartagena, 30202 Cartagena, Murcia, Spain \\ 4 Istituto Nazionale di Astrofisica, Osservatorio Astrofisico di Torino, Strada Osservatorio 20, 10025 Pino Torinese, Italy
}

Received 25 March 2019 / Accepted 6 June 2019

\begin{abstract}
Aims. Our scientific goal is to provide revised membership lists of the $\alpha$ Per, Pleiades, and Praesepe clusters exploiting the second data release of Gaia and produce five-dimensional maps $\left(\alpha, \delta, \pi, \mu_{\alpha} \cos \delta, \mu_{\delta}\right)$ of these clusters.

Methods. We implemented the kinematic method combined with the statistical treatment of parallaxes and proper motions to identify astrometric member candidates of three of the most nearby and best studied open clusters in the sky.

Results. We cross-correlated the Gaia catalogue with large-scale public surveys to complement the astrometry of Gaia with multiband photometry from the optical to the mid-infrared. We identified 517, 1248, and 721 bona fide astrometric member candidates inside the tidal radius of $\alpha$ Per, the Pleiades, and Praesepe, respectively. We cross-matched our final samples with catalogues from previous surveys to address the level of completeness. We update the main physical properties of the clusters, including mean distance and velocity, as well as core, half-mass, and tidal radii. We infer updated ages from the white dwarf members of the Pleiades and Praesepe. We derive the luminosity and mass functions of the three clusters and compare them to the field mass function. We compute the positions in space of all member candidates in the three regions to investigate their distribution in space.

Conclusions. We provide updated distances and kinematics for the three clusters. We identify a list of members in the $\alpha$ Per, Pleiades, and Praesepe clusters from the most massive stars all the way down to the hydrogen-burning limit with a higher confidence and better astrometry than previous studies. We produce complete 5D maps of stellar and substellar bona fide members in these three regions. The photometric sequences derived in several colour-magnitude diagrams represent benchmark cluster sequences at ages from 90 to $600 \mathrm{Myr}$. We note the presence of a stream around the Pleiades cluster extending up to $40 \mathrm{pc}$ from the cluster centre.
\end{abstract}

Key words. stars: low-mass - brown dwarfs - surveys - open clusters and associations: individual: $\alpha$ Persei open clusters and associations: individual: Pleiades - open clusters and associations: individual: Praesepe

\section{Introduction}

Open clusters are small entities of a few hundred to thousands stars loosely bound in a small region of the sky (Lada \& Lada 2003). They represent ideal laboratories to study stellar evolution because their members share the same mean distance, age, and metallicity. The wide range of masses present in these clusters is key to investigating the present-day mass function (Salpeter 1955; Miller \& Scalo 1979; Scalo 1986; Kroupa 2001; Chabrier 2003) and studying processes responsible for the formation of low-mass stars and brown dwarfs (e.g. Chabrier \& Baraffe 2000). More recently, the Kepler K2 mission (Borucki et al. 2010; Howell et al. 2014) has revived the study of open clusters providing thousands of light curves with exquisite photometric precision (Nardiello et al. 2015, 2016; Rebull et al. 2016, 2017; Stauffer et al. 2016; Douglas et al. 2016, 2017) and identified several low-mass eclipsing binaries (Kraus et al. 2015, 2017; David \& Hillenbrand 2015; Lodieu et al. 2015; David et al. 2015, 2016a; Libralato et al. 2016a) and a few transiting planets at different ages (Libralato et al. 2016b; Mann et al. 2016a,b, 2017, 2018; David et al. 2016b,c). These new discoveries help us to constrain evo-

* Full Tables A.1, B.1, and C.1 are only available at the CDS via anonymous ftp to cdsarc.u-strasbg.fr (130.79.128.5) or via http://cdsarc.u-strasbg.fr/viz-bin/qcat?]/A+A/628/A66 lutionary models at different ages and improve our knowledge of planet formation.

Before the advent of the Gaia mission (Gaia Collaboration 2016), the selection of members of open clusters was predominantly limited to ground-based proper motions and multi-band photometry. The second data release of Gaia (Gaia Collaboration 2018 b) offers a unique opportunity to revise the census of membership of the nearest open clusters thanks to exquisite parallaxes, proper motions, and positions with additional radial velocity measurements for a limited sample of (mainly solar-type) stars. With this new dataset in hand we can revise several important questions yet to be solved such as the evolution of rotation as a function of mass and age (see review by Bouvier et al. 2014), the possible age spreads within a given cluster (e.g. Mayne \& Naylor 2008; Parmentier et al. 2014; Niederhofer et al. 2015), and the dynamical masses and ages of low-mass stars used to calibrate model predictions (e.g. Kraus et al. 2015), among others.

In this manuscript, we present an astrometric selection of cluster member candidates in three of the nearest open clusters to the Sun $-\alpha$ Per, the Pleiades, and Praesepe - exploiting the second data release of Gaia (Gaia Collaboration 2018b) and based on our previous work in the Hyades (Lodieu et al. 2019). We provide a revised stellar census in these three regions and a 3D view of the distribution of their members. In Sect. 2 we present the input catalogue of Gaia. In Sect. 3 we describe the three clusters under study and compile lists of previously known 
members to compare with the Gaia catalogues. In Sect. 4 we identify a complete sample of member candidates in $\alpha$ Per, the Pleiades, and Praesepe, address the completeness of previous studies and cleanness of our selection. In Sect. 5 we investigate white dwarfs previously reported as members of these clusters to revise their membership and estimate their ages. In Sect. 6 we derive the stellar luminosity and mass functions and put them into context with previous studies. In Sect. 7 we discuss the spatial distribution of the highest probability stellar member candidates and present the first 3D map of the clusters.

\section{Gaia DR2 sample}

We made use of the Gaia DR2 data (Lindegren et al. 2018; Hambly et al. 2018; Riello et al. 2018; Evans et al. 2018). Our selection criteria were designed to be as inclusive as possible at the beginning and to become more selective later in the process. We limited our original catalogues to parallaxes larger than 1 mas, which is the sole criterion applied to the full Gaia samples. We checked the re-normalised unit weight error (RUWE) defined by Lindegren (2018) for the full files of more than two million sources. We find that $5.1 \%, 4.4 \%$, and $4.6 \%$ of the sources respectively in $\alpha$ Per, the Pleiades, and Praesepe have RUWE values greater than the 1.4 limit set for quality of the sample.

We cross-matched a series of well-known large-scale surveys to complement the Gaia catalogue, keeping all Gaia DR2 sources without a counterpart in those surveys either due to coverage or to brightness or faintness reasons. We considered the following catalogues: the Two Micron All-Sky Survey (2MASS; Cutri et al. 2003; Skrutskie et al. 2006), the Sloan Digital Sky Survey Data Release 12 (SDSS; Abolfathi et al. 2018), the UKIRT Infrared Deep Sky Survey Galactic Clusters Survey (UKIDSS GCS; Lawrence et al. 2007), the Wide-field Infrared Survey Explorer (AllWISE; Wright et al. 2010; Cutri et al. 2014), and the first data release of the Panoramic Survey Telescope and Rapid Response System (PS1; Kaiser et al. 2002; Chambers et al. 2016). We cross-matched the catalogues with a matching radius of 5 arcsec so any unresolved pairs of stars in the large surveys resolved spatially in Gaia were only matched to the closest Gaia source which is also often the brighter of the two possible matches. All matches were made at the epoch of the catalogue by applying the Gaia proper motions to move the possible counterparts in the target catalogue epoch.

\section{Earlier census of stellar and substellar member candidates}

In this section, we describe the main characteristics of each cluster and compiled lists of known members from different proper motion and photometric surveys published over the past decades to identify high-mass stars, low-mass stars, and brown dwarfs.

\subsection{The $\alpha$ Per cluster}

The $\alpha$ Per cluster (Messier 20) is one of the few open star clusters within $200 \mathrm{pc}$ of the Sun and younger than 200 Myr. The cluster is located to the north-east of the F5V supergiant Alpha Persei at a distance originally estimated to $\sim 180 \mathrm{pc}$ (Pinsonneault et al. 1998; Robichon et al. 1999) and later revised to $172.4 \pm 2.7 \mathrm{pc}$ by the re-reduction of the Hipparcos data (van Leeuwen 2009). The cluster members have solar metallicity (Boesgaard \& Friel 1990) and the extinction along the line of sight is estimated to $A_{V}=$ 0.30 mag with a possible differential extinction (Prosser 1992). Its mean proper motion, $\left(\mu_{\alpha} \cos \delta, \mu_{\delta}\right)=(+22.73,-26.51)$ mas $\mathrm{yr}^{-} 1$ (van Leeuwen 2009), lower than the other clusters studied in this paper, and low galactic latitude $\left(b=-7^{\circ}\right)$, makes the membership selection more difficult in Praesepe and the Pleiades. The combination of larger distance but younger age than the Pleiades places the hydrogen-burning limit at similar apparent magnitudes within the reach of Gaia.

We collected a total of 3056 sources from 13 surveys in $\alpha$ Per: Heckmann et al. (1956), Mitchell (1960), Fresneau (1980), Stauffer et al. (1985, 1989a, 1999), Prosser (1992, 1994), Prosser \& Randich (1998), Prosser et al. (1998), Barrado y Navascués et al. (2002), Deacon \& Hambly (2004), Lodieu et al. (2005, 2012a), Zuckerman et al. (2012), and the latest sample of 740 members from Gaia Collaboration (2018a). After removing common sources, we were left with a collection of 1711 candidate members. We did not include the following surveys in our compilation: Kraft (1966), Petrie \& Heard (1969, 1970), Morgan et al. (1971), and Crawford \& Barnes (1974) because we could not retrieve the list of members electronically. We cross-matched this compendium of 1711 members with Gaia DR2, yielding 1429 objects with precise and accurate astrometry. They are distributed in an area of approximately $43-58^{\circ}$ and $45-53^{\circ}$ in right ascension and declination, respectively (Fig. 1). They span the $G$-band magnitude from about 6.5 to 21 mag. In Fig. A.1, we plot these objects in the diagram showing the Gaia magnitude as a function of parallax. We can clearly see the cluster has a mean parallax of about 5.8 mas with a dispersion of $\sim 0.5$ mas, corresponding to $173 \pm 15 \mathrm{pc}$. We also note that a large fraction (about $40 \%$ ) of these 1429 previously known members are most likely non-members because of their inconsistent parallaxes.

\subsection{The Pleiades cluster}

With the Hyades, the Pleiades (M 45) is the best studied northern cluster and qualifies as a benchmark region (Lynga 1981). Numerous multiwavelength surveys have been conducted in the region to study in depth the stellar and substellar members. Pleiades members share a significant common proper motion compared to neighbouring stars with $\left(\mu_{\alpha} \cos \delta, \mu_{\delta}\right) \sim(19.5$,

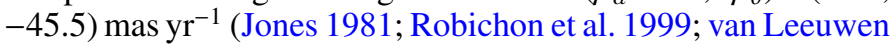
2009). This large mean motion, high galactic latitude $\left(b \sim 24^{\circ}\right)$, and low reddening along the line of sight of the cluster $(E(B-V)=0.03 \mathrm{mag}$; O'dell et al. 1994) ease both astrometric and photometric selections. The cluster is also nearby, with the Hipparcos distance placing the Pleiades at $120.2 \pm 1.9 \mathrm{pc}$ (van Leeuwen 2009) while other works suggest a mean distance of $134 \mathrm{pc}$ with an uncertainty of 5 pc (Johnson 1957; Gatewood et al. 2000; Pinfield et al. 2000; Southworth et al. 2005). The age of the cluster has also been debated in the literature, ranging from 70-80 Myr from the zero age main sequence turn-off and isochrone fitting (Mermilliod 1981; Vandenberg \& Bridges 1984) up to $120-130 \pm 20 \mathrm{Myr}$ as derived from model fitting (Mazzei \& Pigatto 1989; Gossage et al. 2018) and the lithium depletion boundary method (Basri et al. 1996; Stauffer et al. 1998a; Barrado y Navascués et al. 2004) with a revision leading to a possibly younger age (112 $\pm 5 \mathrm{Myr}$; Dahm 2015).

Numerous surveys of distinct sizes and depths have looked at the Pleiades (Stauffer et al. 1991, 1994, 1998b; Simons \& Becklin 1992; Hambly et al. 1993, 1999; Willliams et al. 1996; Cossburn et al. 1997; Zapatero Osorio et al. 1997a,b,c, 1999, 2014a, 2018; Martín et al. 1998; Bouvier et al. 1998; Festin 1998; Pinfield et al. 2000; Adams et al. 2001; Moraux et al. 2001, 2003; Jameson et al. 2002; Dobbie et al. 2002; Deacon \& Hambly 2004; Bihain et al. 2006, 2010; Casewell et al. 2007, 

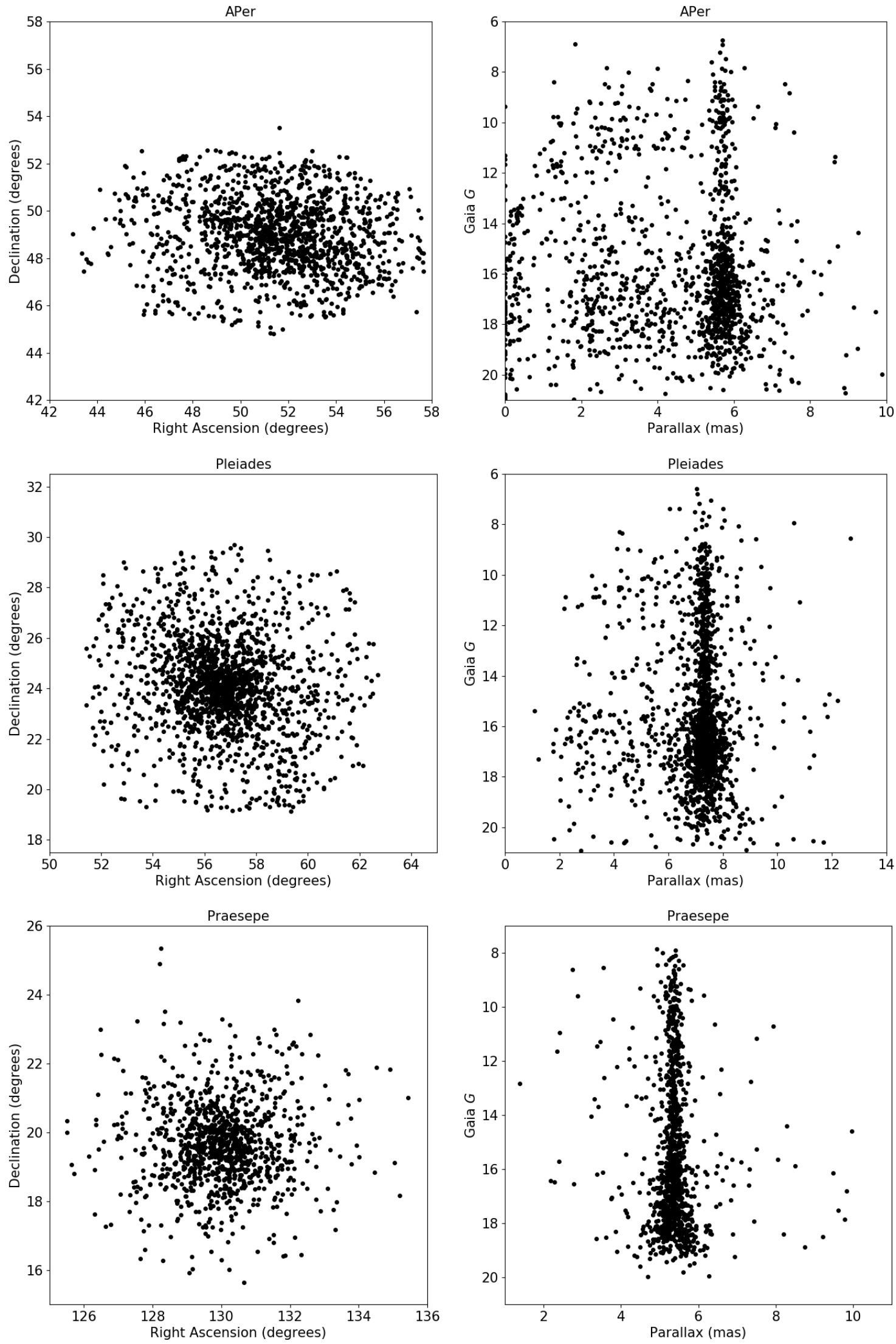

Fig. 1. Left panels: distribution on the sky of selected kinematic member candidates. Right panels: Gaia $G$ magnitude as a function of parallax for previously known members with Gaia parallaxes. From top to bottom, we plot the figures for the $\alpha$ Per, the Pleiades, and Praesepe clusters, respectively. 
2011; Lodieu et al. 2007, 2012b; Bouy et al. 2013; Sarro et al. 2014; Barrado et al. 2016; Gaia Collaboration 2018a). Stauffer et al. (2007) compiled Pleiades members from various sources (see their Table 1) while Sarro et al. (2014), Bouy et al. (2015), and Olivares et al. (2018a) make use of archival datasets to produce the most up-to-date sample of stellar and substellar members. We complemented these two extensive studies with the preliminary Gaia catalogue (Gaia Collaboration 2018a) to produce a full catalogue of 2010 members after removing duplicates (middle row in Fig. 1). We have omitted catalogues of members published before the 1990s, when the first extensive astrometric, photometric, and spectroscopic studies of the Pleiades took place (e.g. Trumpler 1921; Hertzsprung 1947; Artyukhina 1969; Eichhorn et al. 1970; Jones 1973, 1981; Turner 1979; Haro et al. 1982; Stauffer 1982, 1984; van Leeuwen et al. 1986; Jameson \& Skillen 1989; Stauffer et al. 1989b, among others).

\subsection{The Praesepe cluster}

Praesepe (M 44, NGC 2632) is a rich open cluster also known as the Beehive cluster. The cluster is nearby with a mean Hipparcos distance of $181.5 \pm 6.0 \mathrm{pc}$ from the Sun (van Leeuwen 2009). Its proper motion is significant com-

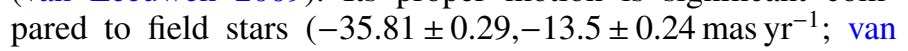
Leeuwen 2009). Moreover, Praesepe might be slightly metal rich $(\mathrm{Fe} / \mathrm{H}=+0.27 \pm 0.10$; Pace et al. 2008) and has negligible reddening. This is an intermediate-age cluster, much older than $\alpha$ Per and the Pleiades but comparable to the Hyades, with estimated ages in the 590-660 Myr range (Vandenberg \& Bridges 1984; Mermilliod 1981; Delorme et al. 2011; Gossage et al. 2018) although older estimates are not discarded (700-900 Myr; Salaris et al. 2004; Bonatto et al. 2004; Brandt \& Huang 2015a; Vandenberg \& Bridges 1984).

Praesepe was first investigated by Klein Wassink $(1924,1927)$ with subsequent studies by Johnson (1952), Artyukhina (1966, 1973), Crawford \& Barnes (1969), Upgren et al. (1979), Weis (1981), Hauck (1981), and Jones \& Cudworth (1983) before exploring lower mass members (Mermilliod et al. 1990; Jones \& Stauffer 1991; Gatewood \& de Jonge 1994; Williams et al. 1994), binarity (Bolte 1991; Mermilliod et al. 1994; Bouvier et al. 2001; Patience et al. 2002; Gillen et al. 2017; Hillenbrand et al. 2018), lithium (King \& Hiltgen 1996; Cummings et al. 2017), and its luminosity and mass functions (Lee et al. 1997; Kraus \& Hillenbrand 2007; Boudreault et al. 2012). The advent of photographic plates with several epochs of observations led to a exhaustive census of stellar members over a large area down to $0.1 M_{\odot}$ (Hambly et al. 1995). The cluster has also been targeted in X-rays by ROSAT (Randich \& Schmitt 1995) and XMM-Newton (Franciosini et al. 2003). The arrival of large-scale surveys and deep optical pencil-beam surveys revealed the low-mass and substellar populations (Adams et al. 2002a; Kraus \& Hillenbrand 2007; Pinfield et al. 1997; Chappelle et al. 2005; González-García et al. 2006; Boudreault et al. 2010, 2012; Baker et al. 2010; Wang et al. 2011, 2014; Boudreault \& Lodieu 2013; Khalaj \& Baumgardt 2013). We compiled all the candidates identified in these surveys and found a total of 6479 sources, 2078 of them being unique among all studies, including the most recent census of members from the Gaia consortium (Gaia Collaboration 2018a).

\section{Selection of member candidates}

We implemented the kinematic procedure described by Perryman et al. (1998) in combination with the Bayesian pro- cedure proposed by Luri et al. (2018) to deal with the proper motions and parallaxes of the Gaia mission. This method determines the barycentre of the cluster and identifies potential members based on their velocities in space (Table 1). For the details of the implementation, we refer the reader to our study of the Hyades cluster (Lodieu et al. 2019).

For completeness, we briefly summarise the steps of the procedure here. We calculated the space velocity of all objects in our initial catalogue following the equations in Perryman et al. (1998). We calculated the cluster barycentre and mean space velocity from the sample of Gaia Collaboration (2018a) and improved it with our final sample. For the sample of Gaia sources with radial velocity measurements, we computed the velocity vector in space. The member candidates statisfy a maximum value of a "degree-of-freedom" parameter, which is described by a confidence region based on the barycentric and velocity positions.

\subsection{Member candidates in $\alpha$ Per}

We derived a mean distance of $177.68 \pm 0.84 \mathrm{pc}$ (Table 1), consistent with the original range of distances (175-190 pc; Pinsonneault et al. 1998; Robichon et al. 1999; Makarov 2006) and slightly further (within $2 \sigma$ ) than the distance from the rereduction of the Hipparcos data (van Leeuwen 2009, 172.4 \pm $2.7 \mathrm{pc}$ ). Our value lies within $3 \sigma$ from the Gaia DR2 value $(174.89 \pm 0.16 \mathrm{pc}$; Gaia Collaboration 2018a). The brightest members of the cluster with $G=6.3-13.7$ mag show a mean lineof-sight velocity of $-0.47 \pm 3.78 \mathrm{~km} \mathrm{~s}^{-1}$ with a mean dispersion of $6.5 \mathrm{~km} \mathrm{~s}^{-1}$, in agreement with earlier studies of bright members (Stauffer et al. 1989a; Prosser 1994; Mermilliod et al. 2008). This translates into a mean cluster velocity of $28.7 \pm 0.5 \mathrm{~km} \mathrm{~s}^{-1}$ (Table 1). The kinematic method returned a total of 3123 member candidates with $G=3.86-21.0 \mathrm{mag}$, corresponding to modeldependent masses of 5.34-0.048 $M_{\odot}$ assuming an age of $90 \mathrm{Myr}$ for $\alpha$ Per.

The core of the cluster was estimated to 1.3 degrees in radius (Artyukhina 1972; Kharchenko et al. 2005), translating into about $4 \mathrm{pc}$ at the distance of the cluster. The core radius is the radius at which the brightness drops to one-half the central value and is equal to 0.64 times the Plummer radius (Plummer 1915). We calculated the density of sources in concentric circles from the centre up to $30 \mathrm{pc}$ with a sampling of $0.4 \mathrm{pc}$ and dividing by the volume (in 3D space). We note that we can not reduce the sampling too much because of the small numbers of sources in the central parsec. We found the best fit of the Plummer model to the density of sources for a core radius of $2.3 \pm 0.3 \mathrm{pc}$, significantly smaller than previous estimates.

The tidal radius is usually considered as the limit where cluster candidates are bound to the cluster, while sources beyond that radius are more affected by the external gravitational field of the galaxy than the cluster dynamics. We calculated the tidal radius of $\alpha$ Per using Eq. (3) in Röser et al. (2011) with values for the constants from Piskunov et al. (2006) $)^{1}$. We summed the masses of all kinematic members up to $15 \mathrm{pc}$ per bins of $1 \mathrm{pc}$ and determined the tidal radius as the radius where the curve derived from Eq. (3) in Röser et al. (2011) crosses the curve defined by the masses of our candidates. We inferred a tidal radius of $\sim 9.5 \mathrm{pc}$ in agreement with the $9.7 \mathrm{pc}$ reported by Makarov (2006). The half-mass radius is $5.6 \mathrm{pc}$ and contains 247 sources. We find 21 and 554 candidate members located within the core and tidal radius of the cluster, respectively. We consider

\footnotetext{
$M_{c}=x l^{3} \cdot / G \times(4 * A *(A-B))$
} 
Table 1. Positional and kinematics data of the Pleiades, Praesepe, and $\alpha$ Per clusters, for which we infer tidal radii of 11.6, 10.7, and 9.5 pc, respectively.

\begin{tabular}{|c|c|c|c|c|c|c|c|c|}
\hline \multirow[t]{2}{*}{ Cluster } & \multirow[t]{2}{*}{ Distance } & \multirow[t]{2}{*}{ Velocity } & \multicolumn{3}{|c|}{$\mathbf{b}_{c}(\mathrm{pc})$} & \multicolumn{3}{|c|}{$\mathbf{v}_{c}\left(\mathrm{~km} \mathrm{~s}^{-1}\right)$} \\
\hline & & & $b_{x}$ & $b_{y}$ & $b_{z}$ & $v_{x}$ & $v_{y}$ & $v_{z}$ \\
\hline & $\mathrm{pc}$ & $\mathrm{km} \mathrm{s}^{-1}$ & $\mathrm{pc}$ & $\mathrm{pc}$ & $\mathrm{pc}$ & $\mathrm{km} \mathrm{s}^{-1}$ & $\mathrm{~km} \mathrm{~s}^{-1}$ & $\mathrm{~km} \mathrm{~s}^{-1}$ \\
\hline$\alpha$ Per & $177.68 \pm 0.84$ & $28.70 \pm 0.52$ & $-148.72 \pm 0.56$ & $95.20 \pm 0.60$ & $-19.77 \pm 0.47$ & $-14.22 \pm 0.49$ & $-23.99 \pm 0.31$ & $-6.77 \pm 0.08$ \\
\hline Pleiades & $135.15 \pm 0.43$ & $32.73 \pm 0.18$ & $-120.25 \pm 0.33$ & $28.94 \pm 0.24$ & $-54.49 \pm 0.23$ & $-7.04 \pm 0.27$ & $-28.53 \pm 0.07$ & $-14.40 \pm 0.13$ \\
\hline Praesepe & $187.35 \pm 3.89$ & $49.18 \pm 2.44$ & $-141.91 \pm 2.59$ & $-69.44 \pm 1.97$ & $100.71 \pm 2.23$ & $-43.44 \pm 1.87$ & $-20.73 \pm 1.02$ & $-10.10 \pm 1.74$ \\
\hline
\end{tabular}

sources beyond the tidal radius unbound to the cluster, yielding to a much lower statistical probability of membership. We limited our study to three times the tidal radius of the cluster, i.e. $28.5 \mathrm{pc}$ where we have 2041 sources. We find 22 pairs of stars with separations of less than 5 arcsec including four marked as duplicated in Gaia DR2. We have 167 sources with radial velocities from Gaia DR2 (about 5.3\%).

We cross-matched the list of previously published members with our full sample and the subsample within the tidal radius with a matching radius of 3 arcsec. The numbers are quoted in Table 2 and provide a level of reliability for earlier studies of the cluster but not strictly completeness due to the variety of areas and depths under study.

We plot the positions of the $\alpha$ Per kinematic member candidates of the cluster within the core, half-mass, and tidal radii and up to $28.5 \mathrm{pc}$ with different colours in right ascension and declination in the top left panel of Fig. A.1 as well as the other diagrams involving parallax, proper motion, and magnitude from Gaia (Fig. A.1). We show various colour-magnitude diagrams with the Gaia filters and photometry from other public surveys in Figs. A.2-A.4.

We observe large numbers of sources much bluer than the cluster sequences in the two faintest magnitude bins when the Gaia $G_{\mathrm{BP}}$ magnitude is involved. This behaviour is reduced when the other two Gaia magnitudes are taken into account and disapear when the Gaia $G$ magnitude is combined with infrared or mid-infrared magnitudes from other public surveys. This peculiarity was already noted in the case of the Alessi 10 cluster (Arenou et al. 2018) and for nearby faint brown dwarfs and the Praesepe cluster (Smart et al. 2019). We confirm a similar behaviour in all three clusters, pointing towards unreliable $G_{\mathrm{BP}}$ magnitudes at the faint end of Gaia when dealing with red sources.

\subsection{Member candidates in the Pleiades}

The distance of the Pleiades has been a subject of debate for the past decades (Pinsonneault et al. 1998; Soderblom et al. 1998; van Leeuwen 2009). The distance has been evaluated with different techniques (isochrone fitting, orbital modeling, trigonometric parallax, moving cluster) usually agreeing on a mean value in the 130-140 pc range (Gatewood et al. 2000; Southworth et al. 2005; Soderblom et al. 2005; Melis et al. 2014; An et al. 2007). On the contrary, analysis of the HiPPARcos data suggested smaller distances close to $120 \mathrm{pc}$ (van Leeuwen et al. 1997; Robichon et al. 1999; van Leeuwen 1999, 2009). Abramson (2018) used Gaia DR2 to provide a new estimate of the parallax ( $7.34 \pm 0.27$ mas), translating into a mean distance of $136.2 \pm 5.0 \mathrm{pc}$. We infer a distance of $135.15 \pm 0.43 \mathrm{pc}$ for the Pleiades from the Bayesian approach of Luri et al. (2018) and kinematic members (Table 1), in agreement with the majority of studies and with the distance from Gaia DR1 (Gaia Collaboration 2016). We find a mean line-of-sight velocity of $5.67 \pm 2.93 \mathrm{~km} \mathrm{~s}^{-1}$ for members of the Pleiades with a dispersion of $4.80 \mathrm{~km} \mathrm{~s}^{-1}$, yielding a mean cluster motion of $32.73 \pm 0.18 \mathrm{~km} \mathrm{~s}^{-1}$. Our values are consistent with earlier studies (Liu et al. 1991; Mermilliod et al. 1997) and the recent determination of Gaia Collaboration (2018a).

The kinematic method returned a total of 2281 member candidates with $G=2.77-21.0 \mathrm{mag}$, corresponding to modeldependent masses of 4.78-0.048 $M_{\odot}$ assuming an age of $125 \mathrm{Myr}$ for the Pleiades. We note that 342 sources have radial velocity measurements from Gaia DR2 $(\sim 15 \%)$. We inferred the core radius following the procedure described in Section 4.1, yielding $2.00 \pm 0.25 \mathrm{pc}$, similar to the intervals of core radii published so far (see Olivares et al. (2018a) for a summary of previous determinations (Pinfield et al. 1998; Raboud \& Mermilliod 1998; Adams \& Myers 2001; Converse \& Stahler 2008, 2010)). We calculated the tidal radius as in Sect. 4.1, deriving $11.6 \mathrm{pc}$, with a total of 1248 sources. The number of sources within the core and half-mass (4.5 pc) radii are 106 and 495, respectively. We limited our search for member candidates up to three times the tidal radius (i.e. $34.8 \mathrm{pc}$ ). We found 36 pairs with separations less than 5 arcsec, including two triple and one quadruple systems with components marked as duplicated in Gaia DR2.

We cross-matched the list of previously published members with our full sample and the subsample within the tidal radius with a matching radius of 3 arcsec. The numbers quoted in Table 3 provide an estimate of the level of completeness of earlier studies of the Pleiades although we should caution that some surveys were dedicated to the faintest members which are too faint for Gaia. We should note that we recovered 2152 sources out of the most complete list of Pleiades members with membership probabilities higher than $75 \%$ (Olivares et al. 2018b). Among those, 1960 are in our input file (91\%) and 1467 (68\%) common to our list of members. We display the members of the Pleiades in the diagrams shown in Figs. B.2-B.4.

\subsection{Member candidates in Praesepe}

We determined a mean distance of $187.35 \pm 3.89 \mathrm{pc}$ for Praesepe (Table 1), which is in agreement with the original estimate from Hipparcos $(188.0 \pm 14 \mathrm{pc}$; van Leeuwen 1999) but is $1 \sigma$ higher than the value inferred from the re-reduction of Hipparcos data $(181.5 \pm 6.0 \mathrm{pc}$; van Leeuwen 2009). Our value also agrees with the values derived from Gaia (186.18 \pm 0.10 ; Gaia Collaboration 2018a). We find a mean line-of-sight velocity of $35.1 \pm 1.7 \mathrm{~km} \mathrm{~s}^{-1}$ with a dispersion of $4.2 \mathrm{~km} \mathrm{~s}^{-1}$, translating into a cluster mean motion of $49.18 \pm 2.44 \mathrm{~km} \mathrm{~s}^{-1}$ (Table 1). Our estimate is comparable to that from a ground-based radial velocity survey (Mermilliod \& Mayor 1999) and the 
Table 2. Summary of the numbers of $\alpha$ Per sources from earlier studies recovered in our full Gaia sample (i.e. up to three times the tidal radius of the cluster).

\begin{tabular}{lcccl}
\hline \hline Method & in Gaia & Kinematic & in $R_{\text {tidal }}$ & Comments \\
\hline Fresneau (1980) & $51 / 56$ & 11 & 7 & Palomar Schmidt astrometric observations \\
Heckmann et al. (1956) & $139 / 140$ & 62 & 49 & See also Mitchell (1960) \\
Prosser (1992) & $79 / 148$ & 57 & 42 & Astrometric+photometric+spectroscopic search \\
Prosser (1994) & $26 / 31$ & 14 & 10 & Photometric, and spectroscopic observations \\
Prosser \& Randich (1998) & $54 / 89$ & 28 & 23 & Follow-up 73 ROSAT sources (Prosser et al. 1996) \\
Prosser et al. (1998) & $45 / 70$ & 23 & 15 & Follow-up 130 ROSAT sources (Prosser et al. 1996) \\
Stauffer et al. (1999) & $24 / 28$ & 14 & 5 & Lithium depletion boundary \\
Barrado y Navascués et al. (2002) & $59 / 101$ & 42 & 24 & Deep wide-field optical survey \\
Deacon \& Hambly (2004) & $289 / 302$ & 192 & 111 & Photographic plates \\
Lodieu et al. (2005) & $1 / 39$ & 1 & 0 & Near-infrared photometric selection \\
Zuckerman et al. (2012) & $135 / 149+29 / 33$ & $116+13$ & $84+2$ & Members+possible members (Tables 1+2) \\
Lodieu et al. (2012a): Table A1: & $367 / 494$ & 241 & 150 & Previous members \\
Lodieu et al. (2012a): Tables B1+C1: & $690 / 728+617 / 685$ & $441+494$ & $240+268$ & New members \\
Gaia Collaboration (2018a) & $740 / 740$ & 739 & 467 & Gaia DR2 \\
\hline
\end{tabular}

Notes. The final list of kinematic member candidates contains 23, 224, 517, 2069 sources in 2.3 (core radius), 5.6 (half-mass radius), 9.5 (tidal radius), and $28.5 \mathrm{pc}$ (3×tidal radius), respectively.

Table 3. Summary of the numbers of Pleiades sources from earlier studies recovered in our full Gaia sample (i.e. up to three times the tidal radius of the cluster).

\begin{tabular}{lcccl}
\hline \hline Method & in Gaia & Kinematic & in $R_{\text {tidal }}$ & Comments \\
\hline Hambly et al. (1993) & $429 / 440$ & 385 & 334 & Photographic plates \\
Zapatero Osorio et al. (1997b) & $3 / 10$ & 1 & 2 & CCD-based $R$, I survey \\
Stauffer et al. (1998b) & $16 / 20$ & 12 & 9 & Lithium depletion boundary; MHO targets \\
Bouvier et al. (1998) & $19 / 26$ & 13 & 8 & Proper motions in Moraux et al. (2001) \\
Moraux et al. (2003) & $103 / 109$ & 93 & 78 & Brown dwarfs and IMF \\
Deacon \& Hambly (2004) & $882 / 916$ & 776 & 642 & Astrometry and photometry of photographic plates \\
Bihain et al. (2006) & $14 / 34$ & 11 & 6 & Deep I + J survey of 1.8 deg ${ }^{2}$ \\
Stauffer et al. (2007) & $1361 / 1416$ & 1129 & 928 & Compilation of earlier studies (their Table 1) \\
Casewell et al. (2007) & $8 / 23$ & 7 & 2 & Deep CFH12K survey combined with UKIDSS \\
Lodieu et al. (2007) & $82 / 116$ & 69 & 46 & UKIDSS GCS DR1 combined with 2MASS \\
Lodieu et al. (2012b) & $1176 / 1314$ & 863 & 700 & Selection in UKIDSS GCS DR9 \\
Zapatero Osorio et al. (2014a) & $5 / 24$ & 4 & 2 & See Zapatero Osorio et al. (2014b) for spectroscopy \\
Bouy et al. (2015) & $1775 / 2010$ & 1421 & 1070 & See also Bouy et al. (2013) and Sarro et al. (2014) \\
Olivares et al. (2018b) & $1960 / 2152$ & 1467 & 1097 & p $\geq 0.75$ from Bayesian hierarchical model \\
Gaia Collaboration (2018a) & $1326 / 1326$ & 1295 & 1087 & Gaia DR2 \\
\hline
\end{tabular}

Notes. We have 106, 495, 1248, and 2195 kinematic member candidates in 3.1, 4.5, 11.6, and 34.8 pc, respectively.

Gaia DR2 mean value $\left(35.64 \pm 0.10 \mathrm{~km} \mathrm{~s}^{-1}\right.$; Gaia Collaboration 2018a).

The kinematic method returned a total of 2200 member candidates with $G=6.13-20.93 \mathrm{mag}$, corresponding to modeldependent masses of 2.6-0.115 $M_{\odot}$ assuming an age of $600 \mathrm{Myr}$ for Praesepe. We note that 223 out of 2200 candidates have Gaia DR2 line-of-sight velocity values (about 10\%). We derived a core radius of $2.6 \pm 0.2 \mathrm{pc}$, smaller than the determination of $3.5 \mathrm{pc}$ by Adams et al. (2002b). We inferred a tidal radius of $10.7 \mathrm{pc}$ with 721 member candidates. We derived a halfmass radius of $5.9 \mathrm{pc}$ with 336 sources, while 77 sources lie within the core radius. We limited our study to $32.1 \mathrm{pc}$ or three times the tidal radius with a total of 1847 sources. We find 23 pairs with separations of less than 5 arcsec, including two triples with the brightest components marked as duplicated in Gaia DR2.

We cross-matched the list of previously published members with our full sample and the subsample within the tidal radius with a matching radius of 3 arcsec. The numbers are quoted in
Table 4 and provide a level of completeness for earlier studies in Praesepe. We display the members of Praesepe in the diagrams displayed in Figs. C.2-C.4.

\section{White dwarfs}

When the age of a star cluster is sufficiently old, at least $~ 50 \mathrm{Myr}$ for a solar composition, some of its high-mass members may have had enough time to evolve down to the white dwarf (WD) cooling sequence. The presence of a few WDs opens the possibility of determining the age of the cluster by evaluating the age of each WD member, as we did in our recent article on the Hyades cluster (Lodieu et al. 2019). We used the same method to determine the age of the clusters under study. Without repeating all the details of this technique already described in Lodieu et al. (2019), we give a summary here of the main steps, focusing on a few differences with respect to that paper.

While the selection of the WDs in each single cluster under study is discussed in the following subsections, we compare the 
Table 4. Summary of the numbers of Praesepe sources from earlier studies recovered in our Gaia sample (i.e. up to three times the tidal radius of the cluster).

\begin{tabular}{lcccl}
\hline \hline Method & in Gaia & Kinematic & in $R_{\text {tidal }}$ & Comments \\
\hline Jones \& Cudworth (1983) & $269 / 282$ & 179 & 144 & Palomar photographic plates \\
Hambly et al. (1995) & $495 / 515$ & 407 & 269 & Photographic plates \\
Pinfield et al. (1997) & $7 / 26$ & 2 & 1 & Follow-up in Hodgkin et al. (1999) and Pinfield et al. (2003) \\
Kraus \& Hillenbrand (2007) & $1083 / 1130$ & 948 & 595 & Census of the cluster \\
González-García et al. (2006) & $2 / 20$ & 0 & 0 & Deep survey focusing on substellar members \\
Boudreault et al. (2010) & $86 / 151$ & 40 & 25 & Wide-field $I+J K$ survey \\
Baker et al. (2010) & $137 / 145$ & 97 & 49 & Cross-match of SDSS and UKIDSS DR7 \\
Wang et al. (2011) & $6 / 59$ & 2 & 1 & Deep pencil-beam survey for brown dwarfs \\
Boudreault et al. (2012) & $980 / 1116$ & 751 & 407 & UKIDSS GCS DR9; see also Boudreault \& Lodieu (2013) \\
Khalaj \& Baumgardt (2013) & $864 / 893$ & 726 & 490 & Cross-match of PPMXL and SDSS \\
Wang et al. (2014) & $991 / 1040$ & 825 & 568 & Cross-match of MASS, PPMXL, and Pan-STARRS \\
Gaia Collaboration (2018a) & $938 / 938$ & 920 & 644 & Gaia DR2 \\
\hline
\end{tabular}

Notes. We have 139, 336, 721, and 1847 kinematic member candidates in 3.5, 5.9, 10.7, and $32.1 \mathrm{pc}$, respectively.

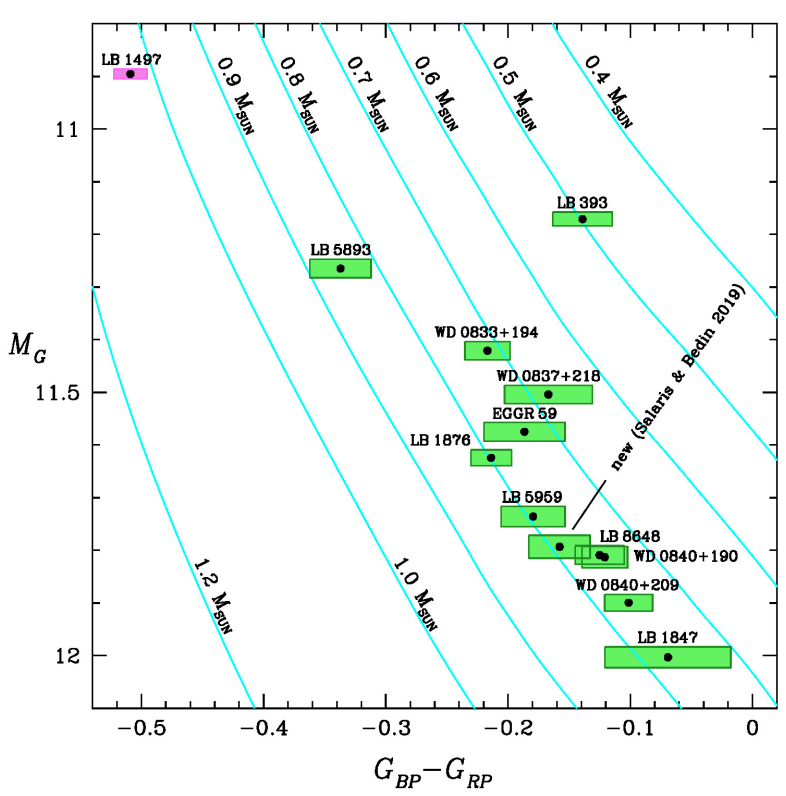

Fig. 2. Absolute magnitude of the white dwarfs in the Pleiades and Praesepe clusters as a function of the $G_{\mathrm{BP}}-G_{\mathrm{RP}}$ colour. The error boxes (pink for the only known WD in the Pleiades, and green for the Praesepe WDs) correspond to the Gaia DR2 photometric (plus parallax) errors. The WD cooling tracks of Bergeron et al. (2011) for various masses are shown in light blue (see text for details).

position of the 13 WDs belonging to the Pleiades and Praesepe clusters in the $\left(G_{\mathrm{BP}}-G_{\mathrm{RP}}, M_{G}\right) \mathrm{HR}$ diagram (Fig. 2) with the cooling tracks of single DA white dwarfs with H-thick envelopes $\left(M_{H} / M_{\star}=10^{-4}\right)$ from Bergeron et al. (2011), see also Tremblay et al. (2011) and references therein. Figure 2 is built considering a reddening $E(B-V)$ equal to 0.045 and 0.027 for the Pleiades and Praesepe, respectively, using Eq. (1) of Gaia Collaboration (2018a) to compute the extinction coefficients. Unlike the relatively bright WDs in the Hyades, for which the photometric errors were very small and negligible in the total error budget (Lodieu et al. 2019), we note that now the photometric errors must be taken into account. From a cubic spline interpolation of the models, we determine the basic stellar parameters of each white dwarf: effective temperature, surface gravity, mass and WD cooling age. After having verified that the effective temperatures and gravities $(\log (g))$ are in good agreement with the known spectroscopic val- ues from the literature, we used the initial-to-final mass relation (IFMR) of El-Badry et al. (2018) to derive the mass of each WD progenitor. Then, for each WD progenitor, we used the Padova evolutionary models for massive stars (Bressan et al. 2012; Tang et al. 2014) with nearly solar abundances $(Z=0.017, Y=0.279)$ to compute the time needed to evolve from the pre-MS to the first thermal pulse in the asymptotic giant branch.

The results of our computations are summarised in Table 5. As in Lodieu et al. (2019), we see that the major contribution to the error budget comes again from the IFMR uncertainty on the initial mass, which propagates into the MS evolutionary time. The errors quoted for the initial masses and the MS evolutionary times correspond to the $95.4 \%$ probability, as described in El-Badry et al. (2018), see also their Fig. 3. The errors quoted for the WD cooling times, effective temperatures, gravities, and masses are conservative values corresponding to the range of the derived parameters from all combinations of the $1 \sigma$ uncertainties plotted in Fig. 2.

Finally it is important to emphasise that the WD ages are very sensitive to the reddening. As we see at the end of Sects. 5.2 and 5.3, small differences in the reddening may cause significant differences in age. When correcting for reddening, WDs are moved toward bluer colors and brighter magnitudes. The strong inclination of the cooling tracks makes the horizontal shift dominant, implying a larger mass, a smaller radius, a lower luminosity and a longer cooling time. This is probably the main limitation of this method, which can produce reliable results only when the reddening is well constrained.

\subsection{White dwarfs in $\alpha$ Per}

Of the 14 white dwarf (WD) candidates proposed by Casewell et al. (2015) in $\alpha$ Per, none have been confirmed to date. Only two of them (AP WD01, AP WD09), both classified as DA by Casewell et al. (2015), are in our list of kinematic candidates but none lies within the tidal radius of the cluster. The closest one, AP WD01, is at $12.6 \mathrm{pc}$ from the cluster centre, while AP WD09 is at $27.2 \mathrm{pc}$. When we compute the total age of these two WDs, i.e. main sequence (MS) age plus WD cooling age, we obtain $130_{-29}^{+30} \mathrm{Myr}$ and $734_{-187}^{+253} \mathrm{Myr}$, respectively (assuming $E(B-$ $V)=0.090$; Gaia Collaboration 2018a). These values, in particular the second, are significantly larger than the ages derived from isochone fitting of the upper main sequence (50-80 Myr; Meynet et al. 1993) and the lithium depletion boundary (85 \pm 10 Myr; Stauffer et al. 1999; Barrado y Navascués et al. 2004), 
Table 5. Derived parameters for the single WD member of the Pleiades (first line) and the 11 WDs of the Praesepe cluster.

\begin{tabular}{|c|c|c|c|c|c|c|c|c|c|c|c|}
\hline Names & Gaia I & $M_{G}$ & $d^{(*)}$ & $\mathrm{SC}$ & $T_{\text {eff }}$ & $\log (g)$ & $M_{\mathrm{WD}}$ & $M_{\mathrm{MS}}$ & $T_{\mathrm{WD}}$ & $T_{\mathrm{MS}}$ & $T_{\text {tot }}$ \\
\hline & & mag & $\mathrm{pc}$ & & K & dex & $M_{\odot}$ & $M_{\odot}$ & ingi & VIyI & Myr \\
\hline LB & 368 & 10.90 & 6.04 & DA & $001_{-1595}^{+2033}$ & $67_{-0.06}^{+0.06}$ & $.05_{-0.04}^{+0.03}$ & $03_{-0.71}^{+1.34}$ & $58_{-2}^{+1}$ & $74_{-27}^{+26}$ & $132_{-27}^{+26}$ \\
\hline LB & 20 & 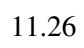 & 2.18 & DA & & & & & & & \\
\hline LB $390=$ & 664325543977630464 & 11.57 & 2.55 & DA & $15428_{-}^{+}$ & $8.20_{-0.09}^{+0.09}$ & $0.74_{-0.06}^{+0.06}$ & $2.96_{-0.34}^{+0.41}$ & & $418_{-133}^{+189}$ & $668_{-133}^{+02}$ \\
\hline WD 08 & 3095377024 & 11.90 & 4.56 & DA & $56_{-473}^{+515}$ & $8.25_{-0.05}^{+0.05}$ & $0.76_{-0.03}^{+0.03}$ & $\begin{array}{l}46 \\
37\end{array}$ & 40 & 36 & $770_{-118}^{+165}$ \\
\hline LB & 66 & & 5.3 & $\mathrm{D}$ & & & & & & & $736_{-123}^{+174}$ \\
\hline LB & 659 & 11.74 & 5 & DA & +833 & $8.29_{-0.07}^{+0.07}$ & $0.79_{-0.04}^{+0.05}$ & $3.31_{-0.39}^{+0.49}$ & & & $608_{-102}^{+138}$ \\
\hline WD & 6610 & 11.81 & 9.49 & DA & $\begin{array}{r}485 \\
471\end{array}$ & $8.23_{-0.05}^{+0.05}$ & 075 & 3.09 & $357_{-19}^{+22}$ & 389 & $746_{-125}^{+177}$ \\
\hline LB & 66 & & 9 & $\mathrm{D}$ & & & & & & $4559_{-127}^{+129}$ & $4743_{-127}^{+129}$ \\
\hline LB 1876 & 661353224747229184 & 11.62 & 13.10 & DA & $16332_{-556}^{+622}$ & $8.30_{-0.05}^{+0.05}$ & $0.80_{-0.03}^{+0.03}$ & $3.30_{-0.39}^{+0.49}$ & $248_{-13}^{+13}$ & $302_{-99}^{+134}$ & $550_{-99}^{+134}$ \\
\hline WD 0833 & 662798086105290112 & 11.42 & 14.17 & DA & $16349_{-609}^{+679}$ & 8.1 & $0.72_{-0.04}^{+0.03}$ & 2.8 & $200_{-12}^{+13}$ & & $664_{-146}^{+206}$ \\
\hline LB 1847 & 6613112 & 12. & 25.61 & $\mathrm{D} A$ & & & & & & & $833_{-114}^{+158}$ \\
\hline WD $0837+218$ & 665139697978259200 & 11.50 & 75.69 & DA & $14825_{-954}^{+1111}$ & $8.11_{-0.09}^{+0.10}$ & $0.68_{-0.06}^{+0.06}$ & $2.52_{-0.28}^{+0.32}$ & $242_{-21}^{+22}$ & $607_{-189}^{+255}$ & $849_{-189}^{+255}$ \\
\hline
\end{tabular}

Notes. ${ }^{(*)}$ Distance from the centre of the cluster.

suggesting that at least APWD09 does not belong to the cluster.

\subsection{White dwarfs in the Pleiades}

In the Pleiades there is only one undisputed white dwarf, LB 1497 (Eggen \& Greenstein 1965), at 6.04 pc from the cluster centre, within the tidal radius but beyond the half-mass radius (Fig. 2; first line of Table 5). Another candidate, GD 50, is ruled out based on its Gaia DR2 distance of $31.21 \pm 0.06 \mathrm{pc}$, which is not compatible with the Pleiades.

From its unique white dwarf LB 1497, and assuming $E(B-$ $V)=0.045$ (Gaia Collaboration 2018a), we derive an age of $132_{-27}^{+26}$ Myr for the Pleiades, which is older than the isochrone fitting (77.6 Myr; Mermilliod 1981), but consistent within $1 \sigma$ to ages from lithium depletion boundary technique $(130 \pm 20 \mathrm{Myr}$; Stauffer et al. 1998a; Barrado y Navascués et al. 2004), model fitting with enhanced overshooting (150 Myr; Mazzei \& Pigatto 1989), and models incorporating rotation (110-160 Myr; Gossage et al. 2018). Just to give an idea of possible systematics due to an incorrect interstellar extinction coefficient, the age would increase to $174_{-35}^{+43}$ Myr if we assume zero reddening.

\subsection{White dwarfs in the Praesepe}

There are 11 known WD candidates in the Praesepe cluster published in the literature (Dobbie et al. 2004, 2006; Casewell et al. 2009). Salaris \& Bedin (2019) confirmed their membership with Gaia DR2 and identified a new member (Gaia DR2 ID 662998983199228032) which is not considered in our analysis given that its spectral class is not known.

For most of the remaining 11 DA WDs, assuming $E(B-V)=$ 0.027 (Gaia Collaboration 2018a), we obtain a total age between 550 and 850 Myr (Table 5). Only one star, LB 5893, is below this range with 304 Myr. Another star, LB 393, returns a completely different age of $4.7 \mathrm{Gyr}$. This star lies at $11.8 \mathrm{pc}$ from the cluster centre, about $1 \mathrm{pc}$ beyond the tidal radius, implying that it might not be bound to Praesepe. Moreover, together with LB 1847, it was excluded from the analysis of Salaris \& Bedin 2019 because of possible problems with $G_{\mathrm{BP}}$ and $G_{\mathrm{RP}}$ photometry (see Salaris \& Bedin 2019, for details). And finally another doubt is raised by the low mass of LB $393\left(0.50 M_{\odot}\right)$ which is close to the typical mass of a hot subdwarf $\left(0.47 M_{\odot}\right)$, that is a star with an initial mass up to $\sim 3 M_{\odot}$, that has lost most of its envelope near the tip of the red giant branch and, after having exhausted its He fuel during the (extreme) horizontal branch, has evolved directly to the WD cooling track, without experiencing a second expansion of the envelope in the asymptotic giant branch (Han et al. 2002). If this were the true evolutionary path of LB 393, its real age could be much less than 4.7 Gyr. When excluding only LB 393, we obtain a mean age of $673_{-39}^{+55} \mathrm{Myr}$ for Praesepe. When excluding the outlier LB 5893 and also excluding LB 1847, which might have the photometric problems stated above, we derive a very similar mean age of $699_{-47}^{+65}$ Myr. Considering only the five WDs within the tidal radius of the cluster $(10.7 \mathrm{pc})$, and excluding LB 5893, we infer a mean age of $706_{-54}^{+76}$ Myr. These estimates are in relatively good agreement with the oldest ages from models incorporating stellar rotation ( 800 Myr; Brandt \& Huang 2015b) but also compatible with the range of ages quoted for Praesepe by other authors (590790 Myr; Mermilliod 1981; Bonatto et al. 2004; Fossati et al. 2008; Delorme et al. 2011; Gossage et al. 2018).

With a zero reddening, the previous age estimates of $673_{-39}^{+55}$, $677_{-46}^{+65}$ and $706_{-54}^{+76} \mathrm{Myr}$ would turn into $799_{-47}^{+64}, 792_{-53}^{+74}$ and $815_{-61}^{+85}$ Myr respectively, with a $\sim 17 \%$ increase.

\section{Luminosity and mass functions}

\subsection{Luminosity functions}

We derive the luminosity functions of the three clusters from our sample of candidates identified astrometrically from the second data release of Gaia. Our samples contain 517, 1248, and 721 member candidates within the tidal radii of the $\alpha$ Per, the Pleiades, and Praesepe clusters. We did not attempt to correct the system luminosity function for binaries and postpone this analysis to later Gaia releases where astrometric parameters of multiple systems will be incorporated.

We display the system luminosity functions, i.e. the number of objects per absolute magnitude bins, with bin width of 1 mag scaled to a volume of one cubic parsec in Fig. 3. We do not apply any correction to the luminosity function because we only consider bound members within the tidal radius of the clusters. Nonetheless, we can discard incompleteness at the very bright 
end or contamination at the faint end where Gaia photometry and astrometry is less reliable, especially in the case of $\alpha$ Per which lies closer to the Galactic plane than the Pleiades and Praesepe.

The luminosity functions of $\alpha$ Per, the Pleiades, and Praesepe show a peak at $M_{G}=9-10 \mathrm{mag}, 11-12 \mathrm{mag}$, and 9-12 mag, respectively. The luminosity functions of the Pleiades and Praesepe increase steadily until they reach a peak and then fall off quickly after; this is due to a combination of smaller numbers of very low-mass members and Gaia detection limits. The luminosity function of $\alpha$ Per displays increases and decreases at bright magnitudes. The peak of the luminosity function appears broader with older ages (Fig. 3).

\subsection{Mass functions}

To convert magnitudes into masses, we need a mass-luminosity relation over a wide mass range, from A-type stars down to the hydrogen-burning limit. To derive the most reliable present-day mass function would require eclipsing binaries with accurate masses and radii over a wide mass range in all three clusters. Unfortunately, such binaries spanning a wide range of properties are not available. Therefore, we adopt a model-dependent massmagnitude relation. We should keep this in mind when interpreting the shape of the mass function.

We combined the Padova isochrones (PARSEC v1.2S plus COLIBRI PR16; Marigo et al. 2008, 2013, 2017; Bressan et al. 2012; Rosenfield et al. 2016) ${ }^{2}$ and the BT-Settl models (Allard et al. 2012; Baraffe et al. 2015) to convert observed magnitudes into masses. We chose ages of $90 \mathrm{Myr}, 125 \mathrm{Myr}$, and $600 \mathrm{Myr}$ for $\alpha$ Per, the Pleiades, and Praesepe as the most common ages quoted in the literature (Sect. 3). We opted to merge the two models to cover the full range of masses where the luminosities at a given mass were the closest. In the case of $\alpha$ Per we kept the Padova and BT-Settl models above and below $1.4 M_{\odot}$ $\left(M_{G} \sim 3.1 \mathrm{mag}\right)$, respectively.

We plot the mass functions in Fig. 4 counting the number of objects per square degree and per bins of 0.1 dex in logarithmic units, assuming the ages quoted above. We included Poisson error bars to each bin of the mass functions. We overplotted the field mass function in logarithmic units (thick red line) from Chabrier (2005), normalised to the numbers of objects per square degree in the bin centred at $0.25 M_{\odot}$. The Pleiades mass function increases steadily up to $\log (M) \sim-0.3 \operatorname{dex}\left(0.5 M_{\odot}\right)$, reaching a plateau followed by a peak at $\operatorname{around} \log (M) \sim-0.8 \mathrm{dex}$ $\left(0.16 M_{\odot}\right)$ before falling down quickly at lower masses and in the substellar regime. In Praesepe, we observe a steep increase from high-mass stars to solar-type stars followed by a plateau with lower mass stars $\left(0.1-0.4 M_{\odot}\right)$. We note the excess of solartype stars $\left(0.5-1.0 M_{\odot}\right.$ and a clear dearth of very low-mass stars (brown dwarfs in Praesepe are too faint for Gaia). In $\alpha$ Per, we observe a slow increase with a broad peak from 0.1 to $0.8 M_{\odot}$ centred on the peak of the field mass function Chabrier (2003). We note a large number of sources at $\log (M) \sim-1.3 \mathrm{dex}$ $\left(0.05 M_{\odot}\right)$ in $\alpha$ Per not present in the other two clusters (Fig. 4). Some of these sources might be photometric non-members based on their location in colour-magnitude diagrams (Figs. A.2-A.4) but the excess might be real and not due to small number statistics because we observe a peak in the luminosity function of $\alpha$ Per not detected in the Pleiades (Fig. 3).

We also investigated the shape of the mass functions as a function of distance from the centre of the cluster. We find that stars contribute in a similar way in the core and half-mass radii

\footnotetext{
2 http://stev.oapd.inaf.it/cgi-bin/cmd
}
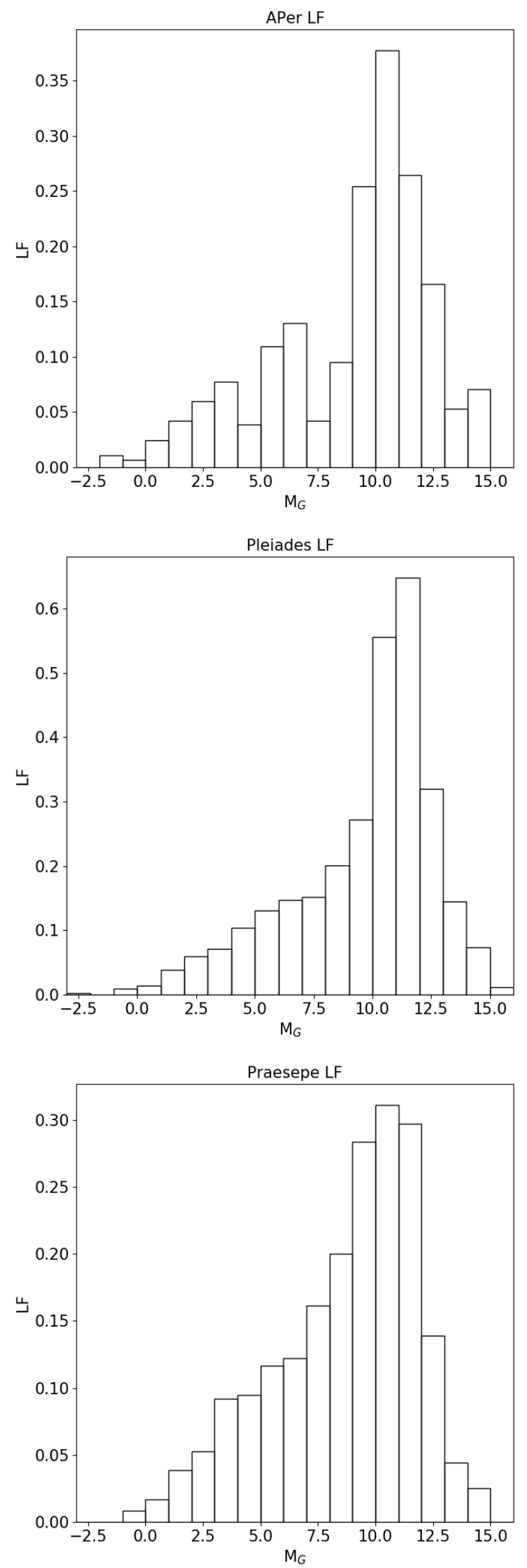

Fig. 3. Luminosity functions, i.e. numbers of objects as a function of absolute $G$ magnitude, within the tidal radius of $\alpha$ Per (bottom), the Pleiades (middle), and Praesepe (top). The number of objects is scaled to a volume of one cubic parsec. 

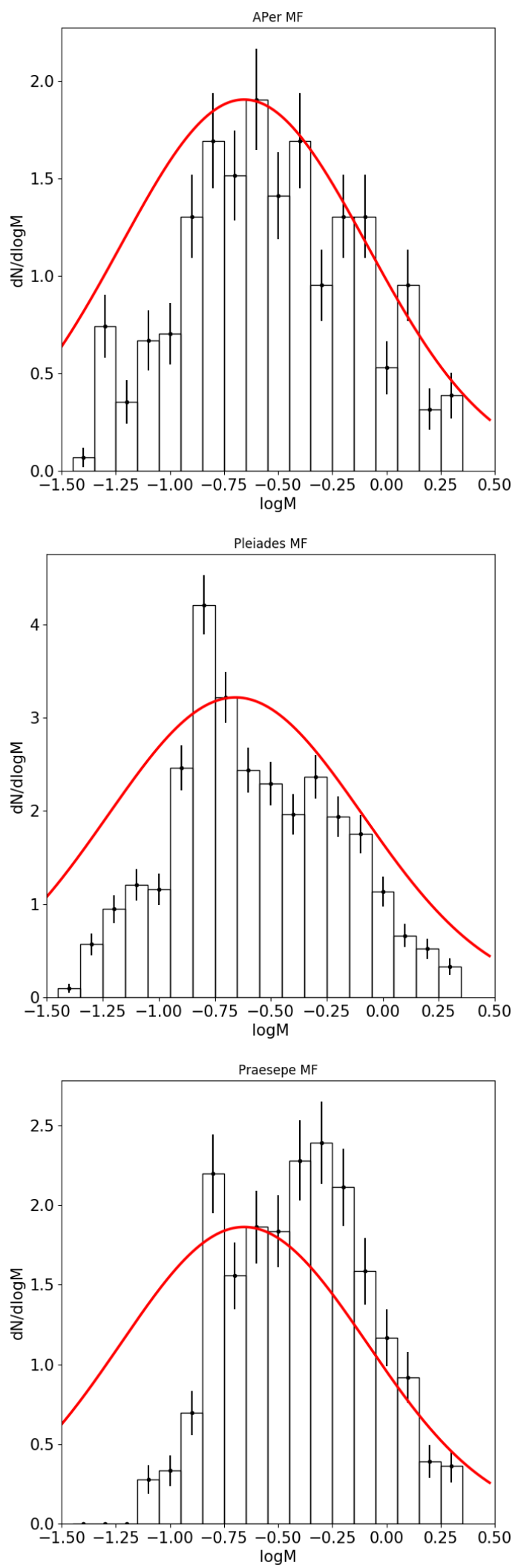

Fig. 4. Mass functions of the $\alpha$ Per (bottom), Pleiades (middle), and Praesepe (top) clusters scaled to a volume of one cubic parsec and a $\log$ Mass bin of 0.1 using isochrones of $90 \mathrm{Myr}, 125 \mathrm{Myr}$, and $600 \mathrm{Myr}$, respectively. Error bars represent the Poisson noise. The field mass function from Chabrier (2003) is overplotted as a thick red line and normalised to the maximum value of the observed mass function at $\sim 0.25 M_{\odot}$. with a dearth of low-mass stars. In the concentric circle delineated by the half-mass and tidal radii, we observe a decrease in high-mass and intermediate-mass stars and an increase in the numbers of low-mass stars, which was expected as the result of mass segregation.

\section{Discussion: 3D maps of the nearest open clusters}

In Fig. 5 we show the positions in space of all member candidates within the tidal radius of the three clusters (black circles) plotted together with all candidates within three times the tidal radius (grey dots). The Galactic system is defined as follows: $x$ is the unit vector towards $(\alpha, \delta)=(0,0), y$ towards $\left(90^{\circ}, 0\right)$, and $\mathrm{z}$ towards $\delta=90^{\circ}$. We observe that Praesepe is the most dispersed of the three clusters most likely due to its older age. Its members seem to disperse in all three directions in a homogeneous manner in the form of a tidal-tail structure. The Pleiades is quite concentrated with about half of its members within the half-mass radius. We detect the presence of a tail-like structure in the $(X, Z)$ direction with an angle of about 30 degrees and extending up to several tens of parsecs from the cluster centre, suggesting that the Pleiades is disintegating and dissipating into the greater stream of the Milky Way (Mädler 1846; Eggen 1975, 1983, 1992, 1995; Dehnen 1998; Liang et al. 2017). In the case of $\alpha$ Per, most of the sources appear concentrated within about $8 \mathrm{pc}$.

We investigated further this tail-like structure of the Pleiades, selecting all candidates within a radius of $50 \mathrm{pc}$ from the centre of the Pleiades. We found 3390 sources satisfying the kinematic criteria. We observed that the tail extends to about $30 \mathrm{pc}$ in the direction $(X, Z)$ and to $50 \mathrm{pc}$ in the opposite direction $(-X,-Z)$. This structure might be related to the Pleiades stream investigated by many authors but with no significant peculiarity detected in terms of abundances and age at the spectral resolution of the Radial Velocity Experiment (RAVE; $R \sim 7500$; Steinmetz et al. 2006), suggesting that it might not be solely the result of cluster disintegration (Famaey et al. 2005, 2008; Antoja et al. 2008, 2012; Zhao et al. 2009; Kushniruk et al. 2017). A better understanding of the origin of the tail-like structures is important to shed light on the formation of the Pleiades and Praesepe clusters and more generally the dynamical history of the Milky Way.

\section{Conclusions}

We presented updated parameters for three nearby open clusters ( $\alpha$ Per, the Pleiades, and Praesepe) and a revised census of member candidates within the tidal radius over the full mass range down to the hydrogen-burning limit.

We summarise the main results of our work as follows:

- We derived a mean distance and velocity of $177.68 \mathrm{pc}$ and $28.70 \mathrm{~km} \mathrm{~s}^{-1}$ for $\alpha$ Per, $135.15 \mathrm{pc}$ and $32.73 \mathrm{~km} \mathrm{~s}^{-1}$ for the Pleiades, and $187.35 \mathrm{pc}$ and $49.18 \mathrm{~km} \mathrm{~s}^{-1}$ for Praesepe. We derived the position of the centre of the cluster and its velocity in space for all regions.

- We identified 517, 1248, and 721 member candidates inside the tidal radius of $\alpha$ Per $(9.5 \mathrm{pc})$, the Pleiades (11.6 pc), and Praesepe $(10.7 \mathrm{pc})$, respectively.

- We inferred ages of $132_{-27}^{+26} \mathrm{Myr}$ and $705_{-54}^{+76} \mathrm{Myr}$ from the WD members within the tidal radius of the Pleiades and Praesepe, respectively, taking into account reddening towards the clusters. None of the WDs previously proposed as members of $\alpha$ Per are recovered as kinematic candidates. 

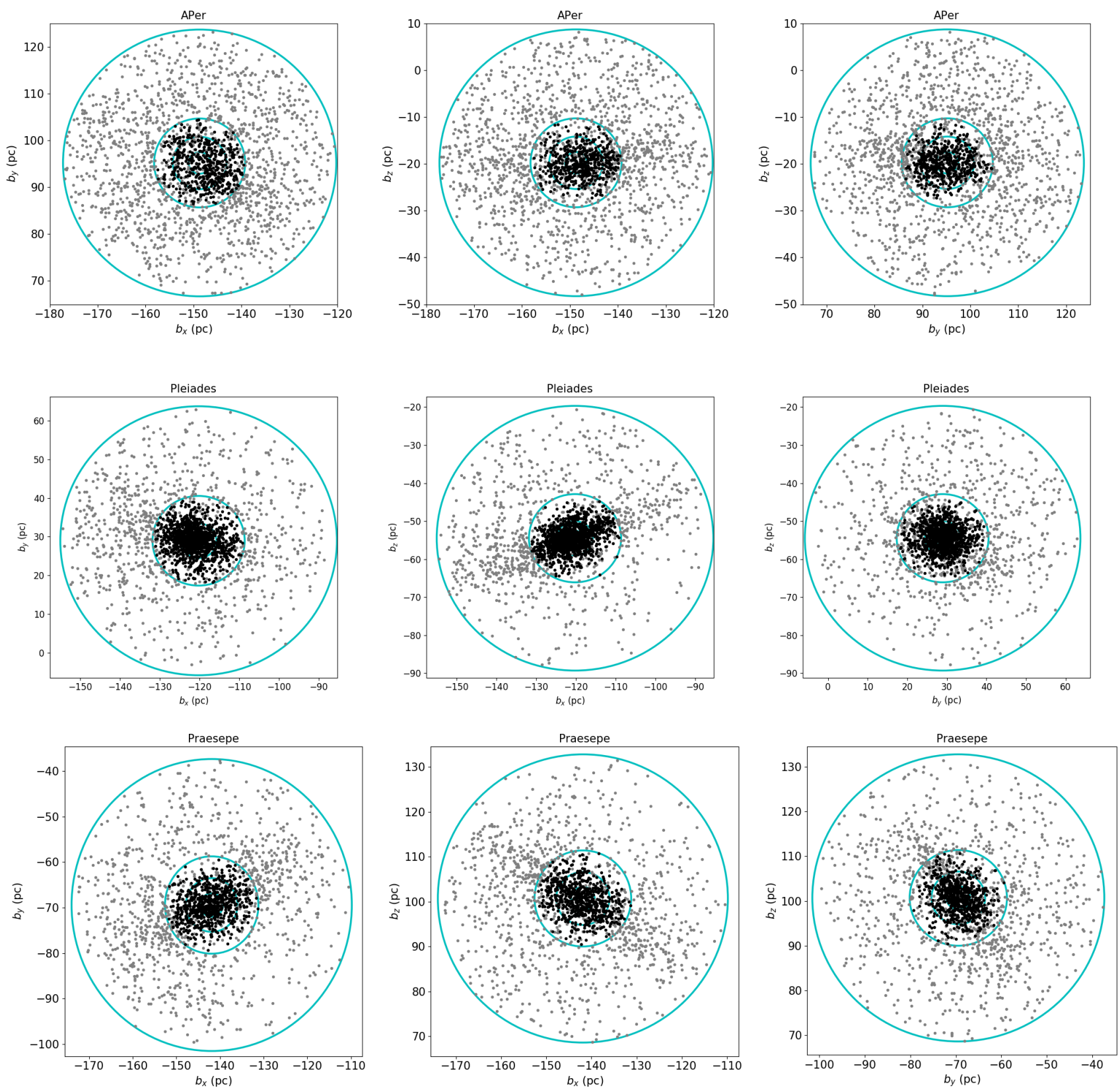

Fig. 5. Positions ( $\mathbf{b}_{c}$ in pc) of the $\alpha$ Per (top row), the Pleiades (middle row), and Praesepe (bottom row) stellar and substellar member candidates in space. Overplotted in cyan are different annuli from the cluster to visualise the distribution and extension of cluster members. Black symbols represent member candidates inside the tidal radius, while grey symbols highlight all candidates in our search up to three times the tidal radius.

- We derived the luminosity and mass functions of the three clusters. We find two peaks at $\sim 0.5$ and $\sim 0.2 M_{\odot}$ in the mass function of the Pleiades. The mass functions of $\alpha$ Per and Praesepe appear relatively flat in the $0.3-1.0 M_{\odot}$ range.

- We calculated the positions in 3D space and find that the members of these three clusters are closely concentrated in a circular manner. We observe the presence of a tail-like structure along the $(X, Z)$ directions in the case of the Pleiades, suggesting that the cluster might soon dissipate. We also see a tail-like structure in all three directions in the case of Praesepe.

- We will publicly release the catalogues of all sources up to three times the tidal radius of the cluster through the Vizier database.

Our study has ignored multiplicity over the full mass range. The next Gaia release should include information on binaries with some preliminary orbits to study the multiplicity as a function of mass and improve the mass determinations for the members of these three clusters with different ages to derive a more accurate mass function. The increment in the total mass of the cluster can increase by double-digit factors depending on the numbers of binaries and mass ratios, as in Praesepe for example (Khalaj \& Baumgardt 2013; Borodina et al. 2019).

Acknowledgements. NL and APG were financially supported by the Spanish Ministry of Economy and Competitiveness (MINECO) under the grants AYA2015-69350-C3-2-P and AYA2015-69350-C3-3-P. RS thanks Pierre Bergeron for having made available, before publication, the WD cooling tracks that include Gaia magnitudes. This research has made use of the Simbad and Vizier databases, operated at the centre de Données Astronomiques de Strasbourg (CDS), and of NASA's Astrophysics Data System Bibliographic Services (ADS). This research has also made use of some of the tools 
developed as part of the Virtual Observatory. This work has made use of data from the European Space Agency (ESA) mission Gaia (https://www . cosmos.esa.int/gaia), processed by the Gaia Data Processing and Analysis Consortium (DPAC, https://www.cosmos.esa.int/web/gaia/dpac/ consortium). Funding for the DPAC has been provided by national institutions, in particular the institutions participating in the Gaia Multilateral Agreement. Funding for the Sloan Digital Sky Survey IV has been provided by the Alfred P. Sloan Foundation, the U.S. Department of Energy Office of Science, and the Participating Institutions. SDSS-IV acknowledges support and resources from the Center for High-Performance Computing at the University of Utah. The SDSS web site is www.sdss.org. SDSS-IV is managed by the Astrophysical Research Consortium for the Participating Institutions of the SDSS Collaboration including the Brazilian Participation Group, the Carnegie Institution for Science, Carnegie Mellon University, the Chilean Participation Group, the French Participation Group, Harvard-Smithsonian Center for Astrophysics, Instituto de Astrofísica de Canarias, The Johns Hopkins University, Kavli Institute for the Physics and Mathematics of the Universe (IPMU)/University of Tokyo, Lawrence Berkeley National Laboratory, Leibniz Institut für Astrophysik Potsdam (AIP), Max-Planck-Institut für Astronomie (MPIA Heidelberg), Max-Planck-Institut für Astrophysik (MPA Garching), Max-Planck-Institut für Extraterrestrische Physik (MPE), National Astronomical Observatories of China, New Mexico State University, New York University, University of Notre Dame, Observatário Nacional/MCTI, The Ohio State University, Pennsylvania State University, Shanghai Astronomical Observatory, United Kingdom Participation Group, Universidad Nacional Autónoma de México, University of Arizona, University of Colorado Boulder, University of Oxford, University of Portsmouth University of Utah, University of Virginia, University of Washington, University of Wisconsin, Vanderbilt University, and Yale University. This publication makes use of data products from the Two Micron All Sky Survey, which is a joint project of the University of Massachusetts and the Infrared Processing and Analysis Center/California Institute of Technology, funded by the National Aeronautics and Space Administration and the National Science Foundation. The UKIDSS project is defined in Lawrence et al. (2007). UKIDSS uses the UKIRT Wide Field Camera (WFCAM; Casali et al. 2007). The photometric system is described in Hewett et al. (2006), and the calibration is described in Hodgkin et al. (2009). The pipeline processing and science archive are described in Irwin et al. (in prep.) and Hambly et al. (2008). This publication makes use of data products from the Wide-field Infrared Survey Explorer, which is a joint project of the University of California, Los Angeles, and the Jet Propulsion Laboratory/California Institute of Technology, and NEOWISE, which is a project of the Jet Propulsion Laboratory/California Institute of Technology. WISE and NEOWISE are funded by the National Aeronautics and Space Administration. The Pan-STARRS1 Surveys (PS1) and the PS1 public science archive have been made possible through contributions by the Institute for Astronomy, the University of Hawaii, the Pan-STARRS Project Office, the Max-Planck Society and its participating institutes, the Max Planck Institute for Astronomy, Heidelberg and the Max Planck Institute for Extraterrestrial Physics, Garching, The Johns Hopkins University, Durham University, the University of Edinburgh, the Queen's University Belfast, the Harvard-Smithsonian Center for Astrophysics, the Las Cumbres Observatory Global Telescope Network Incorporated, the National Central University of Taiwan, the Space Telescope Science Institute, the National Aeronautics and Space Administration under Grant No. NNX08AR22G issued through the Planetary Science Division of the NASA Science Mission Directorate, the National Science Foundation Grant No. AST-1238877, the University of Maryland, Eotvos Lorand University (ELTE), the Los Alamos Nationa Laboratory, and the Gordon and Betty Moore Foundation.

\section{References}

Abolfathi, B., Aguado, D. S., Aguilar, G., et al. 2018, ApJS, 235, 42 Abramson, G. 2018, Res. Notes Am. Astron. Soc., 2, 150

Adams, F. C., \& Myers, P. C. 2001, ApJ, 553, 744

Adams, J. D., Stauffer, J. R., Monet, D. G., Skrutskie, M. F., \& Beichman, C. A 2001, AJ, 121, 2053

Adams, T., Davies, M. B., Jameson, R. F., \& Scally, A. 2002a, MNRAS, 333, 547

Adams, J. D., Stauffer, J. R., Skrutskie, M. F., et al. 2002b, AJ, 124, 1570

Allard, F., Homeier, D., \& Freytag, B. 2012, Roy. Soc. London Philos. Trans. Ser. A, 370, 2765

An, D., Terndrup, D. M., Pinsonneault, M. H., et al. 2007, ApJ, 655, 233

Antoja, T., Figueras, F., Fernández, D., \& Torra, J. 2008, A\&A, 490, 135

Antoja, T., Helmi, A., Bienayme, O., et al. 2012, MNRAS, 426, L1

Arenou, F., Luri, X., Babusiaux, C., et al. 2018, A\&A, 616, A17

Artyukhina, N. M. 1966, Sov. Astron., 43, 132

Artyukhina, N. M. 1969, Sov. Astron., 12, 987

Artyukhina, N. M. 1972, Sov. Astron., 16, 317

Artyukhina, N. M. 1973, Sov. Astron., 17, 65
Baker, D. E. A., Jameson, R. F., Casewell, S. L., et al. 2010, MNRAS, 408, 2457 Baraffe, I., Homeier, D., Allard, F., \& Chabrier, G. 2015, A\&A, 577, A42

Barrado, D., Bouy, H., Bouvier, J., et al. 2016, A\&A, 596, A113

Barrado y Navascués, D., Bouvier, J., Stauffer, J. R., Lodieu, N., \& McCaughrean, M. J. 2002, A\&A, 395, 813

Barrado y Navascués, D., Stauffer, J. R., \& Jayawardhana, R. 2004, ApJ, 614, 386

Basri, G., Marcy, G. W., \& Graham, J. R. 1996, ApJ, 458, 600

Bergeron, P., Wesemael, F., Dufour, P., et al. 2011, ApJ, 737, 28

Bihain, G., Rebolo, R., Béjar, V. J. S., et al. 2006, A\&A, 458, 805

Bihain, G., Rebolo, R., Zapatero Osorio, M. R., Béjar, V. J. S., \& Caballero, J. A. 2010, A\&A, 519, A93

Boesgaard, A. M., \& Friel, E. D. 1990, ApJ, 351, 467

Bolte, M. 1991, ApJ, 376, 514

Bonatto, C., Bica, E., \& Girardi, L. 2004, A\&A, 415, 571

Borodina, O. I., Seleznev, A. F., Carraro, G., \& Danilov, V. M. 2019, ApJ, 874, 127

Borucki, W. J., Koch, D., Basri, G., et al. 2010, Science, 327, 977

Boudreault, S., \& Lodieu, N. 2013, MNRAS, 434, 142

Boudreault, S., Bailer-Jones, C. A. L., Goldman, B., Henning, T., \& Caballero, J. A. 2010, A\&A, 510, A27

Boudreault, S., Lodieu, N., Deacon, N. R., \& Hambly, N. C. 2012, MNRAS, 426, 3419

Bouvier, J., Stauffer, J. R., Martín, E. L., et al. 1998, A\&A, 336, 490

Bouvier, J., Duchêne, G., Mermilliod, J.-C., \& Simon, T. 2001, A\&A, 375, 989

Bouvier, J., Matt, S. P., Mohanty, S., et al. 2014, Protostars and Planets VI, 433

Bouy, H., Bertin, E., Moraux, E., et al. 2013, A\&A, 554, A101

Bouy, H., Bertin, E., Sarro, L. M., et al. 2015, A\&A, 577, A148

Brandt, T. D., \& Huang, C. X. 2015a, ApJ, 807, 58

Brandt, T. D., \& Huang, C. X. 2015b, ApJ, 807, 24

Bressan, A., Marigo, P., Girardi, L., et al. 2012, MNRAS, 427, 127

Casali, M., Adamson, A., Alves de Oliveira, C., et al. 2007, A\&A, 467, 777

Casewell, S. L., Dobbie, P. D., Hodgkin, S. T., et al. 2007, MNRAS, 378, 1131

Casewell, S. L., Dobbie, P. D., Napiwotzki, R., et al. 2009, MNRAS, 395, 1795

Casewell, S. L., Jameson, R. F., Burleigh, M. R., et al. 2011, MNRAS, 412, 2071

Casewell, S. L., Dobbie, P. D., Geier, S., Lodieu, N., \& Hambly, N. C. 2015, MNRAS, 451, 4259

Chabrier, G. 2003, PASP, 115, 763

Chabrier, G. 2005, in The Initial Mass Function 50 Years Later, eds. E. Corbelli,

F. Palla, \& H. Zinnecker, Astrophys. Space Sci. Lib., 327, 41

Chabrier, G., \& Baraffe, I. 2000, ARA\&A, 38, 337

Chambers, K. C., Magnier, E. A., Metcalfe, N., et al. 2016, ArXiv e-prints [arXiv:1612.05560]

Chappelle, R. J., Pinfield, D. J., Steele, I. A., Dobbie, P. D., \& Magazzù, A. 2005, MNRAS, 361, 1323

Converse, J. M., \& Stahler, S. W. 2008, ApJ, 678, 431

Converse, J. M., \& Stahler, S. W. 2010, MNRAS, 405, 666

Cossburn, M. R., Hodgkin, S. T., Jameson, R. F., \& Pinfield, D. J. 1997, MNRAS, 288, L23

Crawford, D. L., \& Barnes, J. V. 1969, AJ, 74, 818

Crawford, D. L., \& Barnes, J. V. 1974, AJ, 79, 687

Cummings, J. D., Deliyannis, C. P., Maderak, R. M., \& Steinhauer, A. 2017, AJ, 153,128

Cutri, R. M., Skrutskie, M. F., van Dyk, S., et al. 2003, 2MASS All Sky Catalog of Point Sources, 2246

Cutri, R. M., Skrutskie, M. F., van Dyk, S., et al. 2014, VizieR Online Data Catalog: II $/ 328$

Dahm, S. E. 2015, ApJ, 813, 108

David, T. J., \& Hillenbrand, L. A. 2015, ApJ, 804, 146

David, T. J., Stauffer, J., Hillenbrand, L. A., et al. 2015, ApJ, 814, 62

David, T. J., Hillenbrand, L. A., Cody, A. M., Carpenter, J. M., \& Howard, A. W. 2016a, ApJ, 816, 21

David, T. J., Conroy, K. E., Hillenbrand, L. A., et al. 2016b, AJ, 151, 112

David, T. J., Hillenbrand, L. A., Petigura, E. A., et al. 2016c, Nat, 534, 658

Deacon, N. R., \& Hambly, N. C. 2004, A\&A, 416, 125

Dehnen, W. 1998, AJ, 115, 2384

Delorme, P., Collier Cameron, A., Hebb, L., et al. 2011, MNRAS, 413, 2218

Dobbie, P. D., Pinfield, D. J., Jameson, R. F., \& Hodgkin, S. T. 2002, MNRAS

335, L79

Dobbie, P. D., Pinfield, D. J., Napiwotzki, R., et al. 2004, MNRAS, 355, L39

Dobbie, P. D., Napiwotzki, R., Burleigh, M. R., et al. 2006, MNRAS, 369, 383

Douglas, S. T., Agüeros, M. A., Covey, K. R., et al. 2016, ApJ, 822, 47

Douglas, S. T., Agüeros, M. A., Covey, K. R., \& Kraus, A. 2017, ApJ, 842, 83

Eggen, O. J. 1975, PASP, 87, 37

Eggen, O. J. 1983, MNRAS, 204, 391

Eggen, O. J. 1992, AJ, 103, 1302

Eggen, O. J. 1995, AJ, 110, 1749

Eggen, O. J., \& Greenstein, J. L. 1965, ApJ, 141, 83 
Eichhorn, H., Googe, W. D., Lukac, C. F., \& Murphy, J. K. 1970, MNRAS, 73, 125

El-Badry, K., Rix, H.-W., \& Weisz, D. R. 2018, ApJ, 860, L17

Evans, D. W., Riello, M., De Angeli, F., et al. 2018, A\&A, 616, A4

Famaey, B., Jorissen, A., Luri, X., et al. 2005, A\&A, 430, 165

Famaey, B., Siebert, A., \& Jorissen, A. 2008, A\&A, 483, 453

Festin, L. 1998, A\&A, 333, 497

Fossati, L., Bagnulo, S., Landstreet, J., et al. 2008, A\&A, 483, 891

Franciosini, E., Randich, S., \& Pallavicini, R. 2003, A\&A, 405, 551

Fresneau, A. 1980, AJ, 85, 66

Gaia Collaboration (Prusti, T., et al.) 2016, A\&A, 595, A1

Gaia Collaboration (Babusiaux, C., et al.) 2018a, A\&A, 616, A10

Gaia Collaboration (Brown, A. G. A., et al.) 2018b, A\&A, 616, A1

Gatewood, G., \& de Jonge, J. K. 1994, ApJ, 428, 166

Gatewood, G., de Jonge, J. K., \& Han, I. 2000, ApJ, 533, 938

Gillen, E., Hillenbrand, L. A., David, T. J., et al. 2017, ApJ, 849, 11

González-García, B. M., Zapatero Osorio, M. R., Béjar, V. J. S., et al. 2006, A\&A, 460, 799

Gossage, S., Conroy, C., Dotter, A., et al. 2018, ApJ, 863, 67

Hambly, N. C., Hawkins, M. R. S., \& Jameson, R. F. 1993, A\&AS, 100, 607

Hambly, N. C., Steele, I. A., Hawkins, M. R. S., \& Jameson, R. F. 1995, MNRAS 273,505

Hambly, N. C., Hodgkin, S. T., Cossburn, M. R., \& Jameson, R. F. 1999, MNRAS, 303, 835

Hambly, N. C., Collins, R. S., Cross, N. J. G., et al. 2008, MNRAS, 384, 637

Hambly, N. C., Cropper, M., Boudreault, S., et al. 2018, A\&A, 616, A15

Han, Z., Podsiadlowski, P., Maxted, P. F. L., Marsh, T. R., \& Ivanova, N. 2002 MNRAS, 336, 449

Haro, G., Chavira, E., \& Gonzalez, G. 1982, Boletin del Instituto de Tonantzintla, 3,3

Hauck, B. 1981, A\&A, 99, 207

Heckmann, O., Dieckvoss, W., \& Kox, H. 1956, Astron. Nachr., 283, 109

Hertzsprung, E. 1947, Annalen van de Sterrewacht te Leiden, 19, A1

Hewett, P. C., Warren, S. J., Leggett, S. K., \& Hodgkin, S. T. 2006, MNRAS, 367,454

Hillenbrand, L. A., Zhang, C., Riddle, R. L., et al. 2018, AJ, 155, 51

Hodgkin, S. T., Pinfield, D. J., Jameson, R. F., et al. 1999, MNRAS, 310, 87

Hodgkin, S. T., Irwin, M. J., Hewett, P. C., \& Warren, S. J. 2009, MNRAS, 394, 675

Howell, S. B., Sobeck, C., Haas, M., et al. 2014, PASP, 126, 398

Jameson, R. F., \& Skillen, I. 1989, MNRAS, 239, 247

Jameson, R. F., Dobbie, P. D., Hodgkin, S. T., \& Pinfield, D. J. 2002, MNRAS 335,853

Johnson, H. L. 1952, ApJ, 116, 640

Johnson, H. L. 1957, ApJ, 126, 121

Jones, B. F. 1973, A\&AS, 9, 313

Jones, B. F. 1981, AJ, 86, 290

Jones, B. F., \& Cudworth, K. 1983, AJ, 88, 215

Jones, B. F., \& Stauffer, J. R. 1991, AJ, 102, 1080

Kaiser, N., Aussel, H., Burke, B. E., et al. 2002, in Survey and Other Telescope Technologies and Discoveries, eds. J. A. Tyson, \& S. Wolff, SPIE Conf. Ser. 4836, 154

Khalaj, P., \& Baumgardt, H. 2013, MNRAS, 434, 3236

Kharchenko, N. V., Piskunov, A. E., Röser, S., Schilbach, E., \& Scholz, R.-D. 2005, A\&A, 438, 1163

King, J. R., \& Hiltgen, D. R. 1996, PASP, 108, 246

Klein Wassink, W. J. 1924, Bull. Astron. Inst. Neth., 2, 183

Klein Wassink, W. J. 1927, Publ. Kapteyn Astron. Lab. Groningen, 41, 1

Kraft, R. P. 1966, AJ, 71, 861

Kraus, A. L., \& Hillenbrand, L. A. 2007, AJ, 134, 2340

Kraus, A. L., Cody, A. M., Covey, K. R., et al. 2015, ApJ, 807, 3

Kraus, A. L., Douglas, S. T., Mann, A. W., et al. 2017, ApJ, 845, 72

Kroupa, P. 2001, MNRAS, 322, 231

Kushniruk, I., Schirmer, T., \& Bensby, T. 2017, A\&A, 608, A73

Lada, C. J., \& Lada, E. A. 2003, ARA\&A, 41, 57

Lawrence, A., Warren, S. J., Almaini, O., et al. 2007, MNRAS, 379, 1599

Lee, S.-W., Sung, H., \& Cho, D.-H. 1997, J. Korean Astron. Soc., 30, 181

Liang, X. L., Zhao, J. K., Oswalt, T. D., et al. 2017, ApJ, 844, 152

Libralato, M., Bedin, L. R., Nardiello, D., \& Piotto, G. 2016a, MNRAS, 456, 1137

Libralato, M., Nardiello, D., Bedin, L. R., et al. 2016b, MNRAS, 463, 1780

Lindegren, L. 2018, Re-normalising the astrometric chi-square in Gaia DR2, Gaia Technical Note: GAIA-C3-TN-LU-LL-124-01

Lindegren, L., Hernández, J., Bombrun, A., et al. 2018, A\&A, 616, A2

Liu, T., Janes, K. A., \& Bania, T. M. 1991, ApJ, 377, 141

Lodieu, N., McCaughrean, M. J., Barrado y Navascués, D., Bouvier, J., \& Stauffer, J. R. 2005, A\&A, 436, 853

Lodieu, N., Dobbie, P. D., Deacon, N. R., et al. 2007, MNRAS, 380, 712
Lodieu, N., Deacon, N. R., Hambly, N. C., \& Boudreault, S. 2012a, MNRAS, 426, 3403

Lodieu, N., Deacon, N. R., \& Hambly, N. C. 2012 b, MNRAS, 2699

Lodieu, N., Burgasser, A. J., Pavlenko, Y., \& Rebolo, R. 2015, A\&A, 579, A58

Lodieu, N., Smart, R. L., Perez-Garrido, A., \& Silvotti, R. 2019, A\&A, 623, A35

Luri, X., Brown, A. G. A., Sarro, L. M., et al. 2018, A\&A, 616, A9

Lynga, G. 1981, Astron. Data Center Bull., 1, 90

Mädler, J. H. 1846, Astron. Nachr., 24, 213

Makarov, V. V. 2006, AJ, 131, 2967

Mann, A. W., Gaidos, E., Mace, G. N., et al. 2016a, ApJ, 818, 46

Mann, A. W., Newton, E. R., Rizzuto, A. C., et al. 2016b, AJ, 152, 61

Mann, A. W., Gaidos, E., Vanderburg, A., et al. 2017, AJ, 153, 64

Mann, A. W., Vanderburg, A., Rizzuto, A. C., et al. 2018, AJ, 155, 4

Marigo, P., Girardi, L., Bressan, A., et al. 2008, A\&A, 482, 883

Marigo, P., Bressan, A., Nanni, A., Girardi, L., \& Pumo, M. L. 2013, MNRAS, 434, 488

Marigo, P., Girardi, L., Bressan, A., et al. 2017, ApJ, 835, 77

Martín, E. L., Zapatero Osorio, M. R., \& Rebolo, R. 1998, in Brown Dwarfs and Extrasolar Planets, eds. R. Rebolo, E. L. Martín, \& M. R. Zapatero Osorio, ASP Conf. Ser., 134, 507

Mayne, N. J., \& Naylor, T. 2008, MNRAS, 386, 261

Mazzei, P., \& Pigatto, L. 1989, A\&A, 213, L1

Melis, C., Reid, M. J., Mioduszewski, A. J., Stauffer, J. R., \& Bower, G. C. 2014, Science, 345, 1029

Mermilliod, J. C. 1981, A\&A, 97, 235

Mermilliod, J. C., \& Mayor, M. 1999, A\&A, 352, 479

Mermilliod, J.-C., Weis, E. W., Duquennoy, A., \& Mayor, M. 1990, A\&A, 235, 114

Mermilliod, J.-C., Duquennoy, A., \& Mayor, M. 1994, A\&A, 283, 515

Mermilliod, J.-C., Bratschi, P., \& Mayor, M. 1997, A\&A, 320, 74

Mermilliod, J. C., Queloz, D., \& Mayor, M. 2008, A\&A, 488, 409

Meynet, G., Mermilliod, J., \& Maeder, A. 1993, A\&AS, 98, 477

Miller, G. E., \& Scalo, J. M. 1979, ApJS, 41, 513

Mitchell, R. I. 1960, ApJ, 132, 68

Moraux, E., Bouvier, J., \& Stauffer, J. R. 2001, A\&A, 367, 211

Moraux, E., Bouvier, J., Stauffer, J. R., \& Cuillandre, J.-C. 2003, A\&A, 400, 891

Morgan, W. W., Hiltner, W. A., \& Garrison, R. F. 1971, AJ, 76, 242

Nardiello, D., Bedin, L. R., Nascimbeni, V., et al. 2015, MNRAS, 447, 3536

Nardiello, D., Libralato, M., Bedin, L. R., et al. 2016, MNRAS, 455, 2337

Niederhofer, F., Georgy, C., Bastian, N., \& Ekström, S. 2015, MNRAS, 453, 2070

O'dell, M. A., Hendry, M. A., \& Collier Cameron, A. 1994, MNRAS, 268, 18

Olivares, J., Moraux, E., Sarro, L. M., et al. 2018a, A\&A, 612, A70

Olivares, J., Sarro, L. M., Moraux, E., et al. 2018b, A\&A, 617, A15

Pace, G., Pasquini, L., \& François, P. 2008, A\&A, 489, 403

Parmentier, G., Pfalzner, S., \& Grebel, E. K. 2014, ApJ, 791, 132

Patience, J., Ghez, A. M., Reid, I. N., \& Matthews, K. 2002, AJ, 123, 1570

Perryman, M. A. C., Brown, A. G. A., Lebreton, Y., et al. 1998, A\&A, 331, 81

Petrie, R. M., \& Heard, J. F. 1969, Publ. Dominion Astrophys. Obs. Victoria, 13 329

Petrie, R. M., \& Heard, J. F. 1970, Publ. Dominion Astrophys. Obs. Victoria, 13, 329

Pinfield, D. J., Hodgkin, S. T., Jameson, R. F., Cossburn, M. R., \& von Hippel, T. 1997, MNRAS, 287, 180

Pinfield, D. J., Jameson, R. F., \& Hodgkin, S. T. 1998, MNRAS, 299, 955

Pinfield, D. J., Hodgkin, S. T., Jameson, R. F., et al. 2000, MNRAS, 313, 347

Pinfield, D. J., Dobbie, P. D., Jameson, R. F., et al. 2003, MNRAS, 342, 1241

Pinsonneault, M. H., Stauffer, J., Soderblom, D. R., King, J. R., \& Hanson, R. B. 1998, ApJ, 504, 170

Piskunov, A. E., Kharchenko, N. V., Röser, S., Schilbach, E., \& Scholz, R.-D 2006, A\&A, 445, 545

Plummer, H. C. 1915, MNRAS, 76, 107

Prosser, C. F. 1992, AJ, 103, 488

Prosser, C. F. 1994, AJ, 107, 1422

Prosser, C. P., \& Randich, S. 1998, Astron. Nachr., 319, 201

Prosser, C. F., Randich, S., Stauffer, J. R., Schmitt, J. H. M. M., \& Simon, T. 1996, AJ, 112, 1570

Prosser, C. P., Randich, S., \& Simon, T. 1998, Astron. Nachr., 319, 215

Raboud, D., \& Mermilliod, J.-C. 1998, A\&A, 329, 101

Randich, S., \& Schmitt, J. H. M. M. 1995, A\&A, 298, 115

Rebull, L. M., Stauffer, J. R., Bouvier, J., et al. 2016, AJ, 152, 114

Rebull, L. M., Stauffer, J. R., Hillenbrand, L. A., et al. 2017, ApJ, 839, 92

Riello, M., De Angeli, F., Evans, D. W., et al. 2018, A\&A, 616, A3

Robichon, N., Arenou, F., Mermilliod, J.-C., \& Turon, C. 1999, A\&A, 345, 471

Rosenfield, P., Marigo, P., Girardi, L., et al. 2016, ApJ, 822, 73

Röser, S., Schilbach, E., Piskunov, A. E., Kharchenko, N. V., \& Scholz, R.-D. 2011, A\&A, 531, A92

Salaris, M., \& Bedin, L. R. 2019, MNRAS, 483, 3098 
Salaris, M., Weiss, A., \& Percival, S. M. 2004, A\&A, 414, 163

Salpeter, E. E. 1955, ApJ, 121, 161

Sarro, L. M., Bouy, H., Berihuete, A., et al. 2014, A\&A, 563, A45

Scalo, J. M. 1986, Fundam. Cosmic Phys., 11, 1

Simons, D. A., \& Becklin, E. E. 1992, ApJ, 390, 431

Skrutskie, M. F., Cutri, R. M., Stiening, R., et al. 2006, AJ, 131, 1163

Smart, R. L., Marocco, F., Sarro, L. M., et al. 2019, MNRAS, 485, 4423

Soderblom, D. R., King, J. R., Hanson, R. B., et al. 1998, ApJ, 504, 192

Soderblom, D. R., Nelan, E., Benedict, G. F., et al. 2005, AJ, 129, 1616

Southworth, J., Maxted, P. F. L., \& Smalley, B. 2005, A\&A, 429, 645

Stauffer, J. R. 1982, AJ, 87, 1507

Stauffer, J. R. 1984, ApJ, 280, 189

Stauffer, J. R., Hartmann, L. W., Burnham, J. N., \& Jones, B. F. 1985, ApJ, 289, 247

Stauffer, J. R., Hartmann, L. W., \& Jones, B. F. 1989a, ApJ, 346, 160

Stauffer, J., Hamilton, D., Probst, R., Rieke, G., \& Mateo, M. 1989b, ApJ, 344, L21

Stauffer, J., Klemola, A., Prosser, C., \& Probst, R. 1991, AJ, 101, 980

Stauffer, J. R., Hamilton, D., \& Probst, R. G. 1994, AJ, 108, 155

Stauffer, J. R., Schultz, G., \& Kirkpatrick, J. D. 1998a, ApJ, 499, 199

Stauffer, J. R., Schild, R., Barrado y Navascues, D., et al. 1998b, ApJ, 504, 805

Stauffer, J. R., Barrado y Navascués, D., Bouvier, J., et al. 1999, ApJ, 527, 219

Stauffer, J. R., Hartmann, L. W., Fazio, G. G., et al. 2007, ApJS, 172, 663

Stauffer, J., Rebull, L., Bouvier, J., et al. 2016, AJ, 152, 115

Steinmetz, M., Zwitter, T., Siebert, A., et al. 2006, AJ, 132, 1645

Tang, J., Bressan, A., Rosenfield, P., et al. 2014, MNRAS, 445, 4287

Tremblay, P.-E., Ludwig, H.-G., Steffen, M., Bergeron, P., \& Freytag, B. 2011, A\&A, 531, L19
Trumpler, R. J. 1921, Lick Obs. Bull., 10, 110

Turner, D. G. 1979, PASP, 91, 642

Upgren, A. R., Weis, E. W., \& Deluca, E. E. 1979, AJ, 84, 1586

Vandenberg, D. A., \& Bridges, T. J. 1984, ApJ, 278, 679

van Leeuwen, F. 1999, A\&A, 341, L71

van Leeuwen, F. 2009, A\&A, 497, 209

van Leeuwen, F., Alphenaar, P., \& Brand, J. 1986, A\&AS, 65, 309

van Leeuwen, F., Hansen Ruiz, C. S., et al. 1997, in Hipparcos - Venice '97, eds.

R. M. Bonnet, E. Høg, P. L. Bernacca, et al., ESA Spec. Publ., 402, 689

Wang, W., Boudreault, S., Goldman, B., et al. 2011, A\&A, 531, A164

Wang, P. F., Chen, W. P., Lin, C. C., et al. 2014, ApJ, 784, 57

Weis, E. W. 1981, PASP, 93, 437

Williams, S. D., Stauffer, J. R., Prosser, C. F., \& Herter, T. 1994, PASP, 106, 817 Williams, D. M., Boyle, R. P., Morgan, W. T., et al. 1996, ApJ, 464, 238 Wright, E. L., Eisenhardt, P. R. M., Mainzer, A. K., et al. 2010, AJ, 140, 1868

Zapatero Osorio, M. R., Martín, E. L., \& Rebolo, R. 1997a, A\&A, 323, 105 Zapatero Osorio, M. R., Rebolo, R., \& Martín, E. L. 1997b, A\&A, 317, 164 Zapatero Osorio, M. R., Rebolo, R., Martín, E. L., et al. 1997c, ApJ, 491, L81 Zapatero Osorio, M. R., Rebolo, R., Martín, E. L., et al. 1999, A\&AS, 134, 537 Zapatero Osorio, M. R., Gálvez Ortiz, M. C., Bihain, G., et al. 2014a, A\&A, 568 , A77

Zapatero Osorio, M. R., Béjar, V. J. S., Martín, E. L., et al. 2014b, A\&A, 572, A67

Zapatero Osorio, M. R., Béjar, V. J. S., Lodieu, N., \& Manjavacas, E. 2018, MNRAS, 475, 139

Zhao, J., Zhao, G., \& Chen, Y. 2009, ApJ, 692, L113

Zuckerman, B., Melis, C., Rhee, J. H., Schneider, A., \& Song, I. 2012, ApJ, 752, 58 


\section{Appendix A: Tables and diagrams for $\alpha$ Per}

We make public via CDS/Vizier the full table of $\alpha$ Per candidate members after applying the kinematic analysis described in Sect. 4. The full catalogue contains 2041 sources within $28.5 \mathrm{pc}$ (three times the size of the tidal radius) with Gaia DR2 data and photometry from several large-scale surveys. Below we show a subset with a limited numbers of columns for space reasons, including source identifiers, coordinates, proper motions, parallaxes, and $G$ magnitudes.
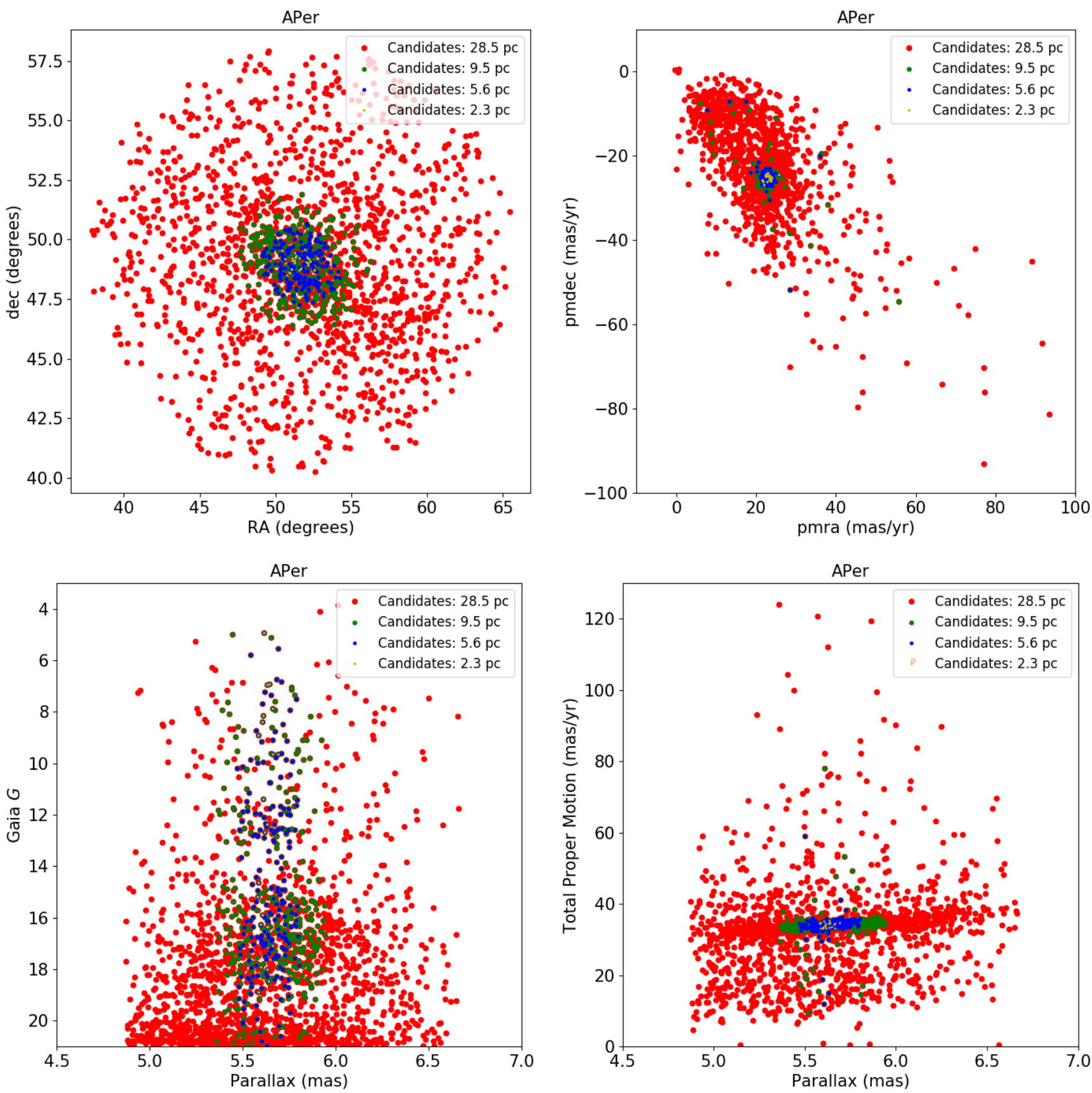

Fig. A.1. Top left: (RA,Dec) diagram showing the position of $\alpha$ Per member candidates. Samples at different distances from the cluster centre are highlighted with distinct colours and sizes. Top right: vector point diagram. Bottom left: Gaia magnitude vs. parallax. Bottom right: total proper motion as a function of parallax. 

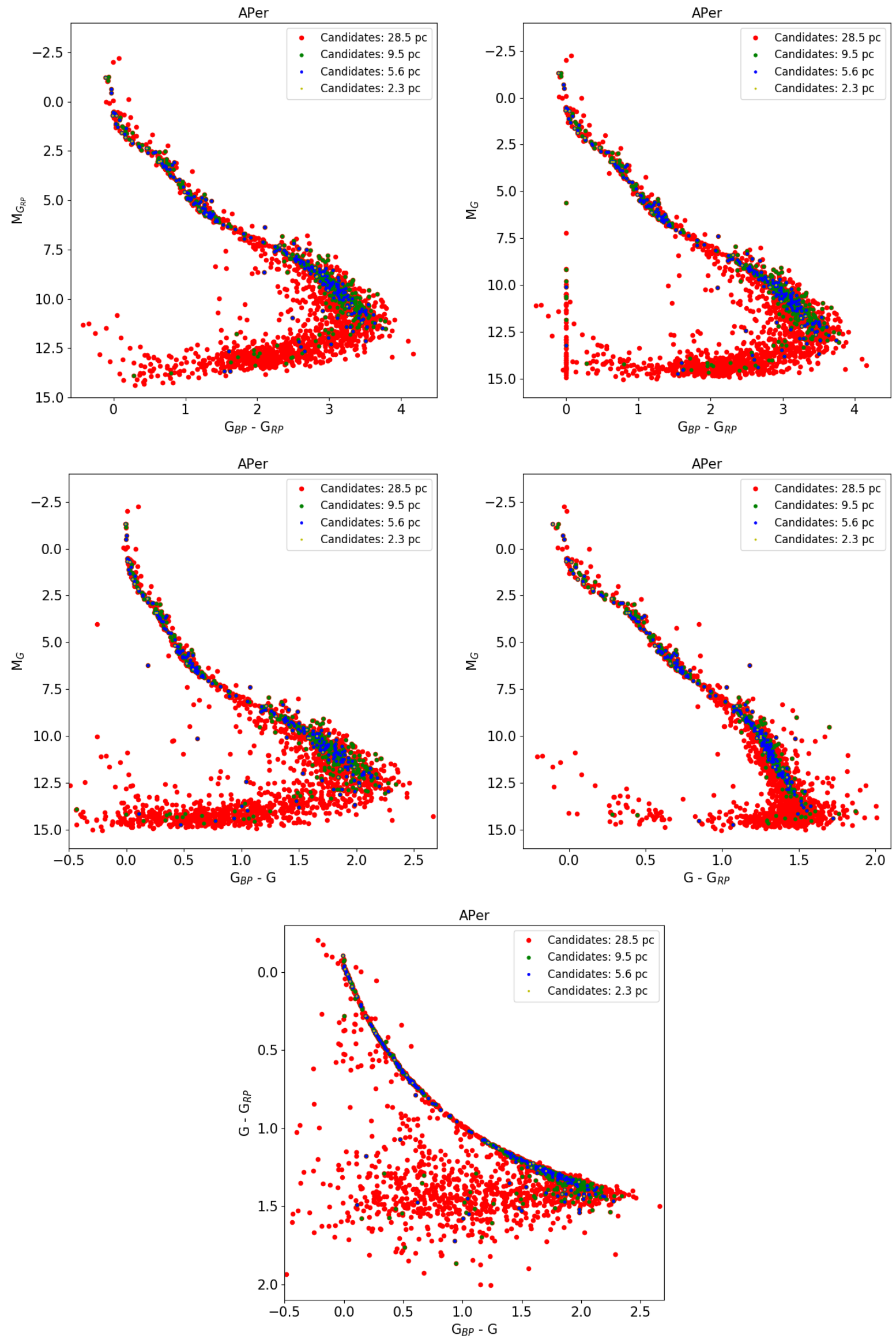

Fig. A.2. Colour-magnitude (top and middle rows) and colour-colour (bottom panel) diagrams with Gaia photometry only for all $\alpha$ Per candidates within a radius of $28.5^{\circ}$ from the cluster centre. We added the full Gaia catalogue in a large region around $\alpha$ Per (small grey dots). 
N. Lodieu et al.: A 5D view of the $\alpha$ Per, Pleiades, and Praesepe clusters
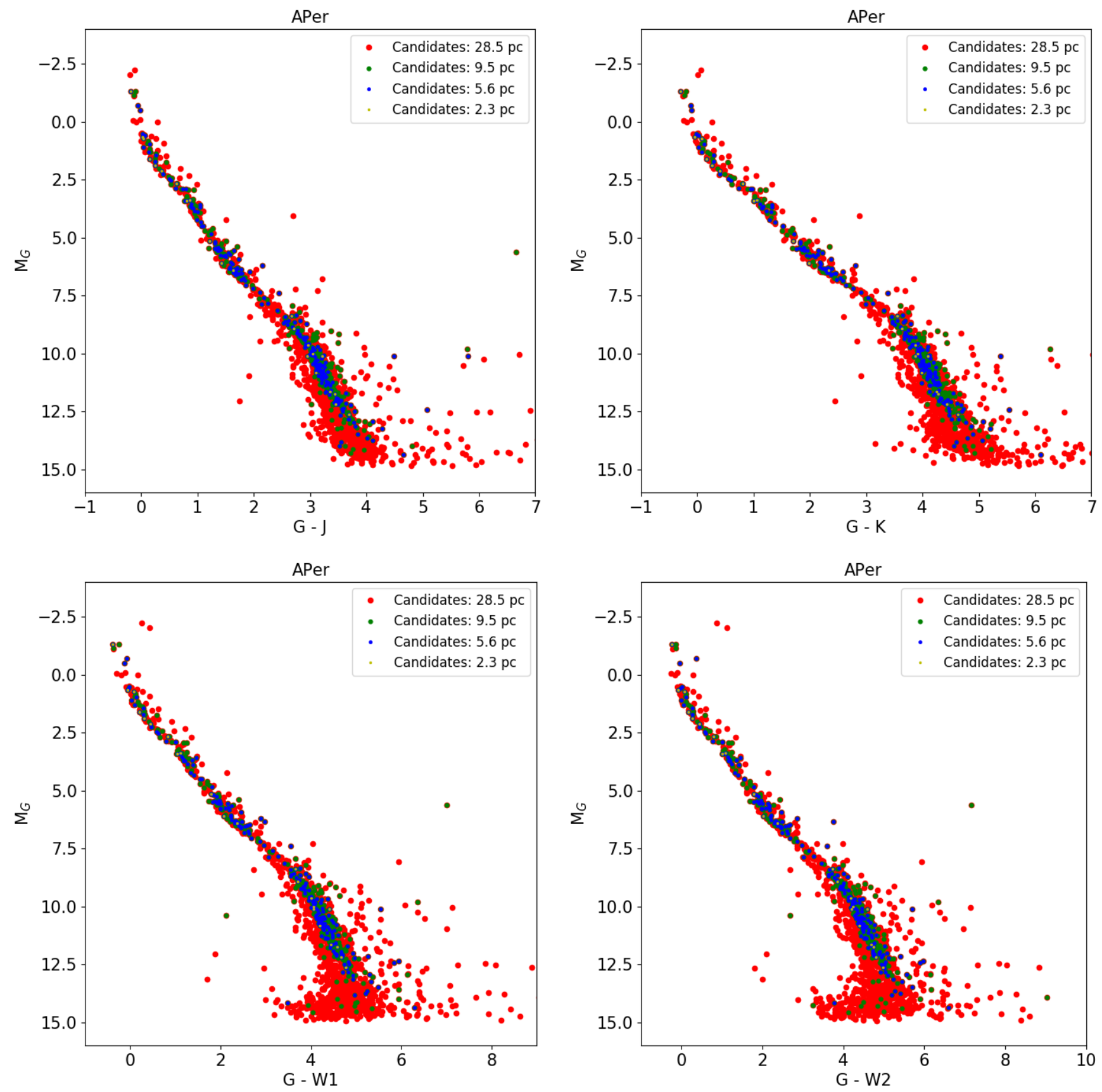

Fig. A.3. Colour-magnitude diagrams for $\alpha$ Per combining the Gaia magnitude with infrared photometry from 2MASS $\left(J+K_{s}\right)$ and AllWISE $(W 1+W 2)$. Symbols are as in Fig. A.2. 

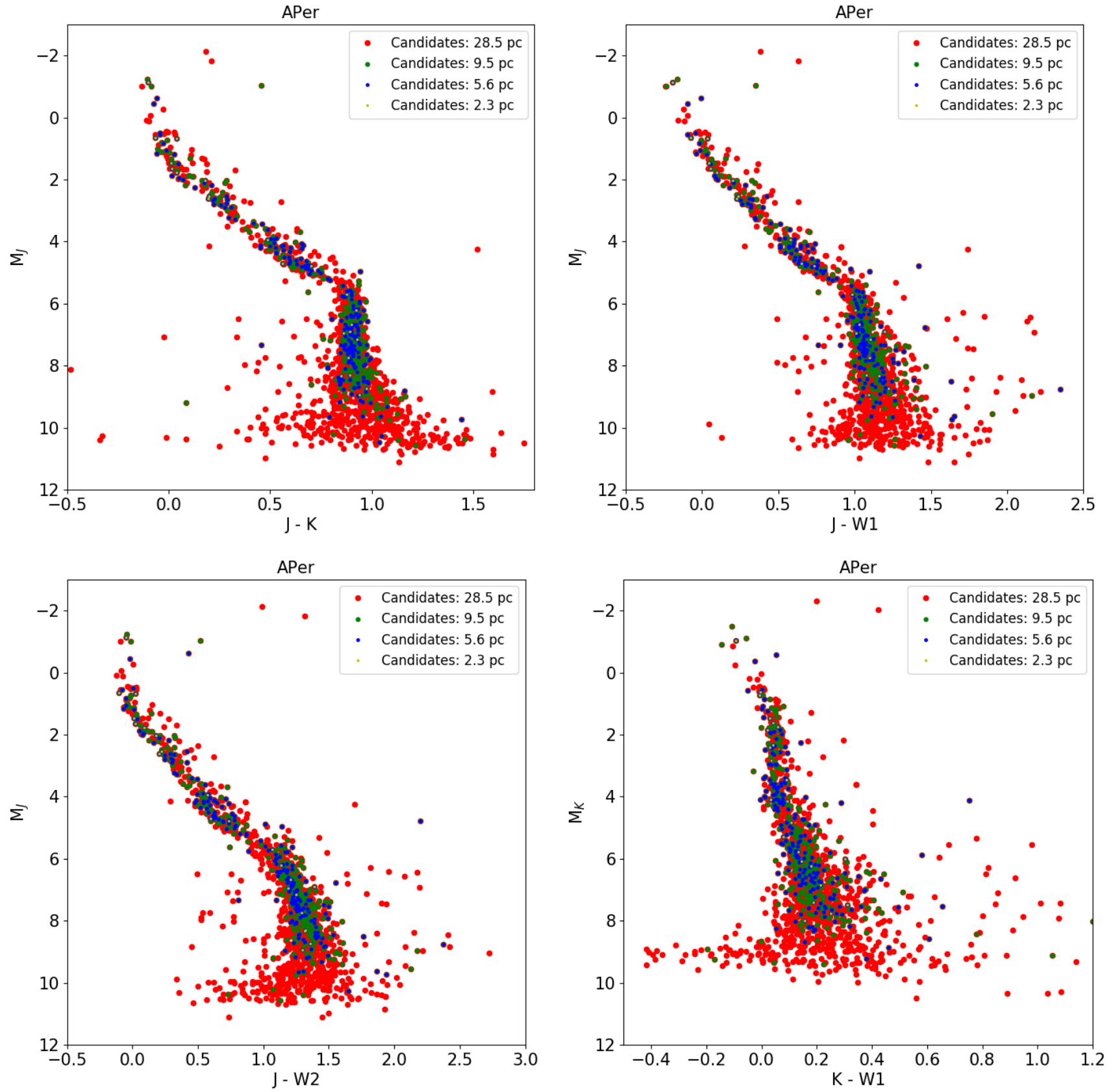

Fig. A.4. Colour-magnitude diagrams for $\alpha$ Per with non-Gaia photometric passbands. Symbols are as in Fig. A.2.

Table A.1. Catalogue of $\alpha$ Per member candidates located within the tidal radius of the cluster.

\begin{tabular}{|c|c|c|c|c|c|c|c|c|c|c|c|c|c|c|c|c|}
\hline SourceID & RA & Dec & pmRA & Dec & Plx & $G$ & $b_{x}$ & $b_{y}$ & $b_{z}$ & $v_{x}$ & $v_{y}$ & $v_{z}$ & $d_{\text {centre }}$ & $c$ & Iass & RV \\
\hline & $\operatorname{deg}$ & $\operatorname{deg}$ & $\operatorname{mas} \mathrm{yr}^{-1}$ & mas $\mathrm{yr}^{-1}$ & mas & mag & $\mathrm{pc}$ & $\mathrm{pc}$ & $\mathrm{pc}$ & $\mathrm{m} \mathrm{s}^{-1}$ & $\mathrm{~km} \mathrm{~s}^{-1}$ & $\mathrm{~km} \mathrm{~s}^{-1}$ & $\mathrm{pc}$ & & $M_{\odot}$ & $\mathrm{m} \mathrm{s}^{-1}$ \\
\hline 455075360092433920 & 34.813289504406 & 52.493073691625 & 27.646 & -19.901 & 5.846 & 16.533 & -122.27 & 117.16 & -24.09 & - & - & - & 0.387 & 34.644 & 0.286 & - \\
\hline 4550 & 35.0102520 & 664085 & 564 & 69 & 5.650 & 20.310 & 26.70 & 121.17 & -24.31 &  & - & - & 13.402 & 34.346 & 0.058 & - \\
\hline 3552425 & 35.095645199238 & 48.516677 & 27.676 & -19. & 5.940 & 15.840 & -122.03 & 110 & -34.36 & 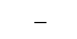 & - & - & 1.227 & 34.172 & 0.391 & - \\
\hline 36467200 & 35.202350 & 49.437643 & 36.234 & -24.237 & 5.819 & 17.689 & -124.45 & 113.99 & -32.46 & - & - & - & 19.035 & 33.215 & 0.168 & - \\
\hline 355706893648353280 & 35.216075974941 & 50.602272518113 & 15.200 & -8.305 & 5.782 & 19.424 & -124.88 & 115.99 & -29.41 & - & - & - & 13.275 & 33.071 & 0.075 & - \\
\hline 354025942235087872 & 35.272857635410 & 47.078048854761 & 22.771 & -2.507 & 5.072 & 20.892 & -143.60 & 127.53 & -44.64 & - & - & - & 13.241 & 41.109 & 0.049 & - \\
\hline 257580321299859968 & 68.322204619080 & 48.021785586470 & 20.927 & -27.332 & 5.885 & 17.712 & -155.67 & 68.12 & 0.25 & - & - & - & 5.921 & 34.384 & 0.167 & - \\
\hline 258437635428197632 & 68.330791212726 & 48.879728011747 & 22.456 & -24.707 & 5.881 & 20.040 & -155.00 & 69.86 & 2.00 & - & - & - & 6.117 & 33.992 & 0.062 & - \\
\hline 257268682770968960 & 68.353707068218 & 46.624841369087 & 17.959 & & 5.857 & 17.395 & -157.60 & 65.59 & -2.53 & - & - & - & 2.246 & 35.395 & 0.188 & - \\
\hline 271026936190122112 & 68.428008586545 & 51.372991304681 & 6.104 & -10.112 & 5.522 & 20.742 & -162.55 & 79.45 & 7.62 & - & - & - & 10.021 & 34.489 & "" & - \\
\hline 271250446285756928 & 68.529601743168 & 52.049391468516 & 22.941 & -38.325 & 6.016 & 19.207 & -148.56 & 74.08 & 8.46 & - & - & - & 5.695 & 35.251 & 0.082 & - \\
\hline 272473279313927424 & 69.157470869484 & 51.414405941179 & 12.183 & -32.031 & 5.810 & 16.682 & -154.82 & 74.75 & 8.34 & - & - & - & 19.058 & 35.288 & 0.267 & - \\
\hline
\end{tabular}

Notes. We list only a subsample of member candidates. The full table is available at the CDS. 


\section{Appendix B: Tables and diagrams for Pleiades}

We make public via CDS/Vizier the full table of Pleiades candidate members after applying the kinematic analysis described in Sect. 4. The full catalogue contains 2195 sources located within three times the tidal radius $(34.8 \mathrm{pc})$ with Gaia DR2 data and photometry from several large-scale surveys. Below we show a subset with a limited numbers of columns for space reasons, including source identifiers, coordinates, proper motions, parallaxes, and $G$ magnitudes.
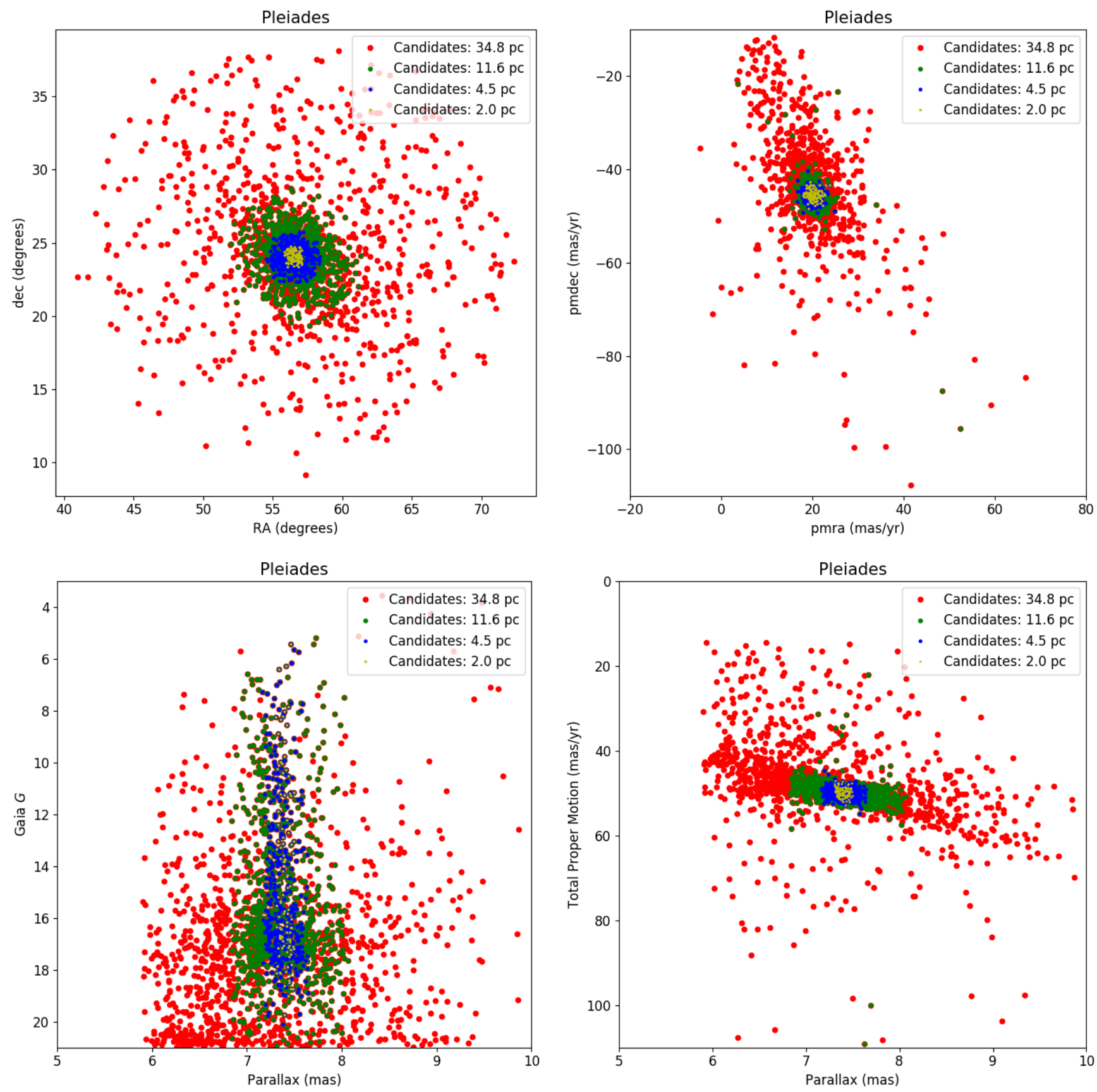

Fig. B.1. Top left: (RA,Dec) diagram showing the position of the Pleiades member candidates. Samples at different distances from the cluster centre are highlighted with distinct colours and sizes. Top right: vector point diagram. Bottom left: Gaia magnitude vs parallax. Bottom right: total proper motion as a function of parallax. 

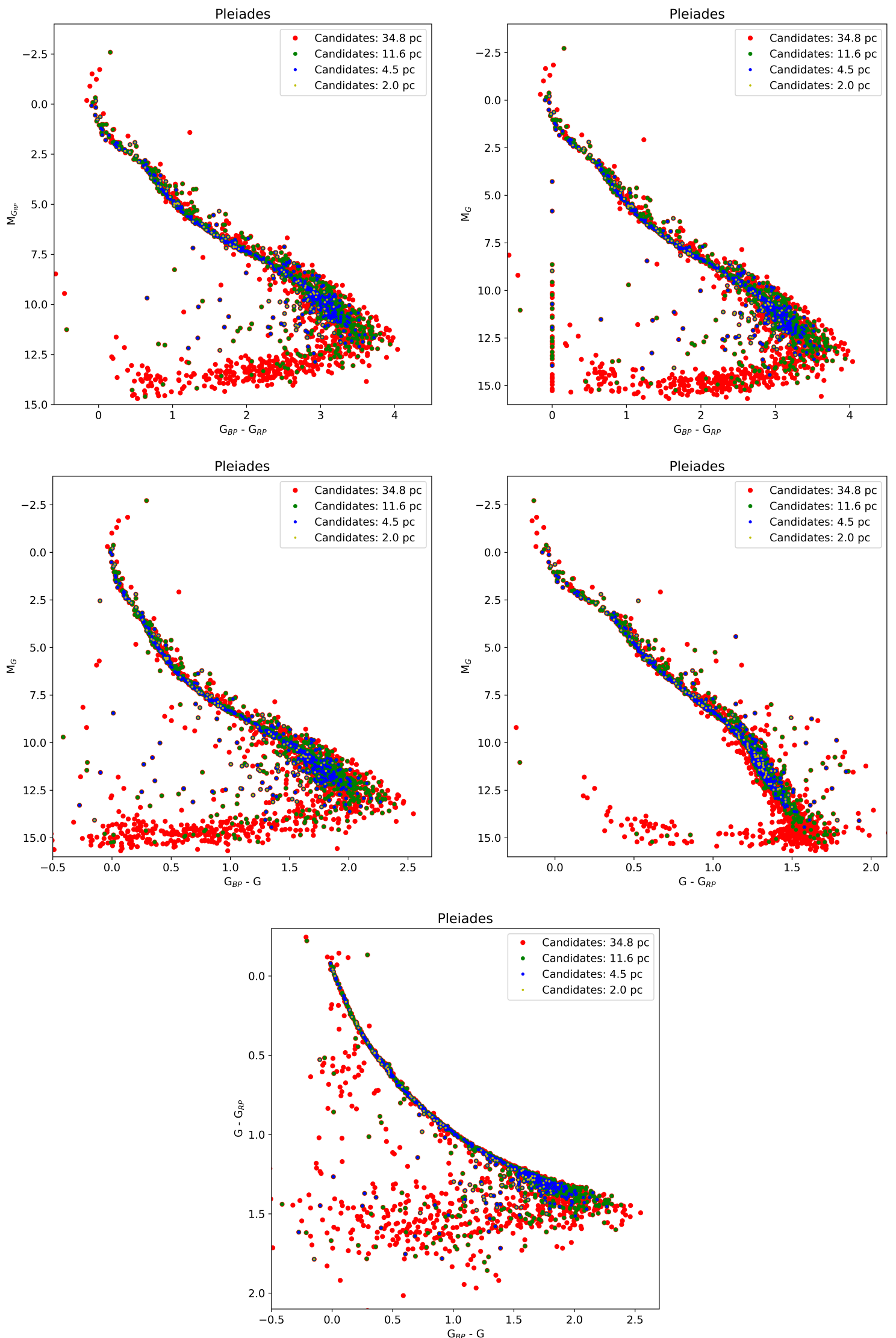

Fig. B.2. Colour-magnitude (top and middle rows) and colour-colour (bottom panel) diagrams with Gaia photometry only for all Pleiades candidates within a radius of $\sim 35^{\circ}$ from the cluster centre. We added the full Gaia catalogue in a large region around Pleiades (small grey dots). 
N. Lodieu et al.: A 5D view of the $\alpha$ Per, Pleiades, and Praesepe clusters
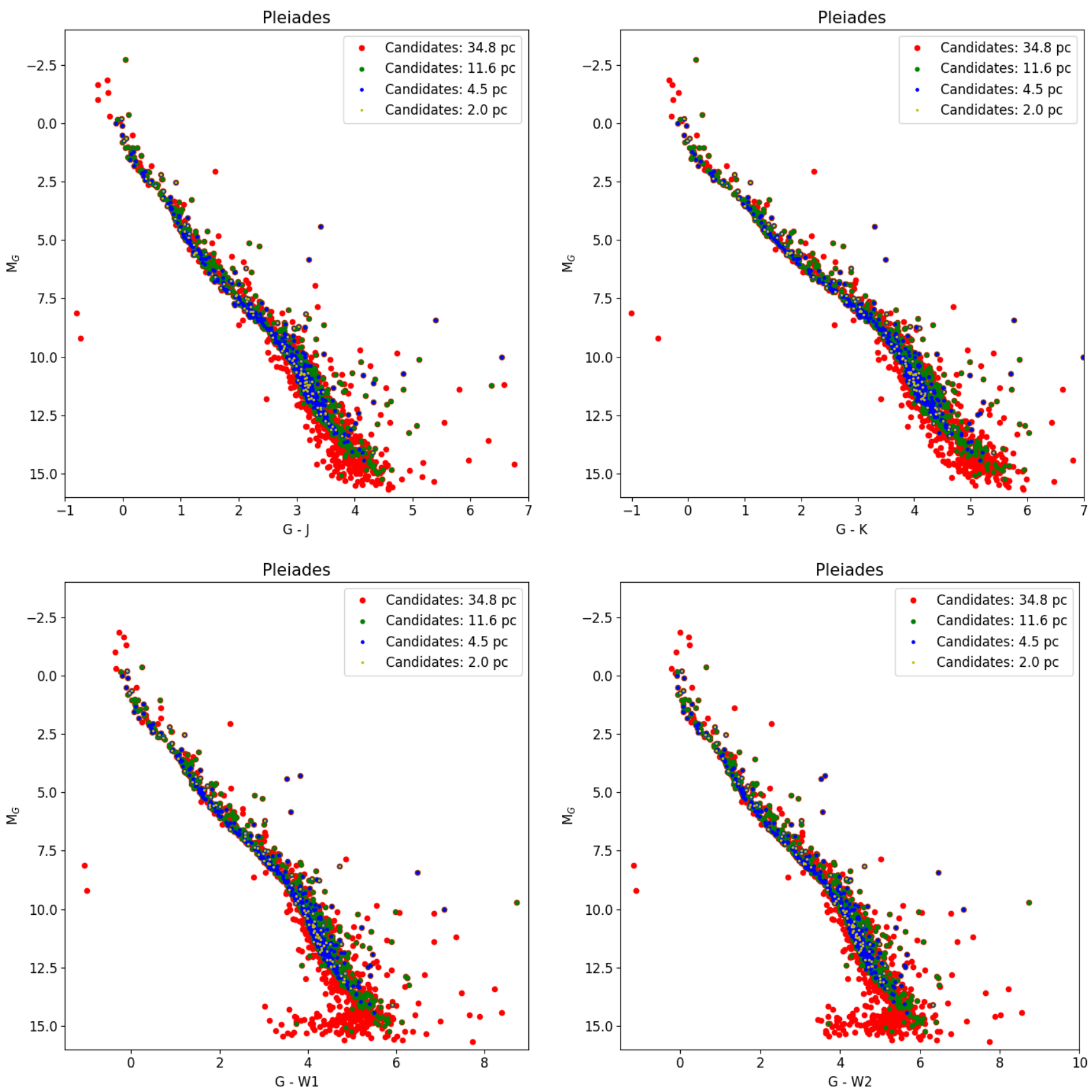

Fig. B.3. Colour-magnitude diagrams for the Pleiades combining the Gaia magnitude with infrared photometry from $2 \mathrm{MASS}\left(J+K_{s}\right)$ and AllWISE $(W 1+W 2)$. Symbols are as in Fig. B.2. 

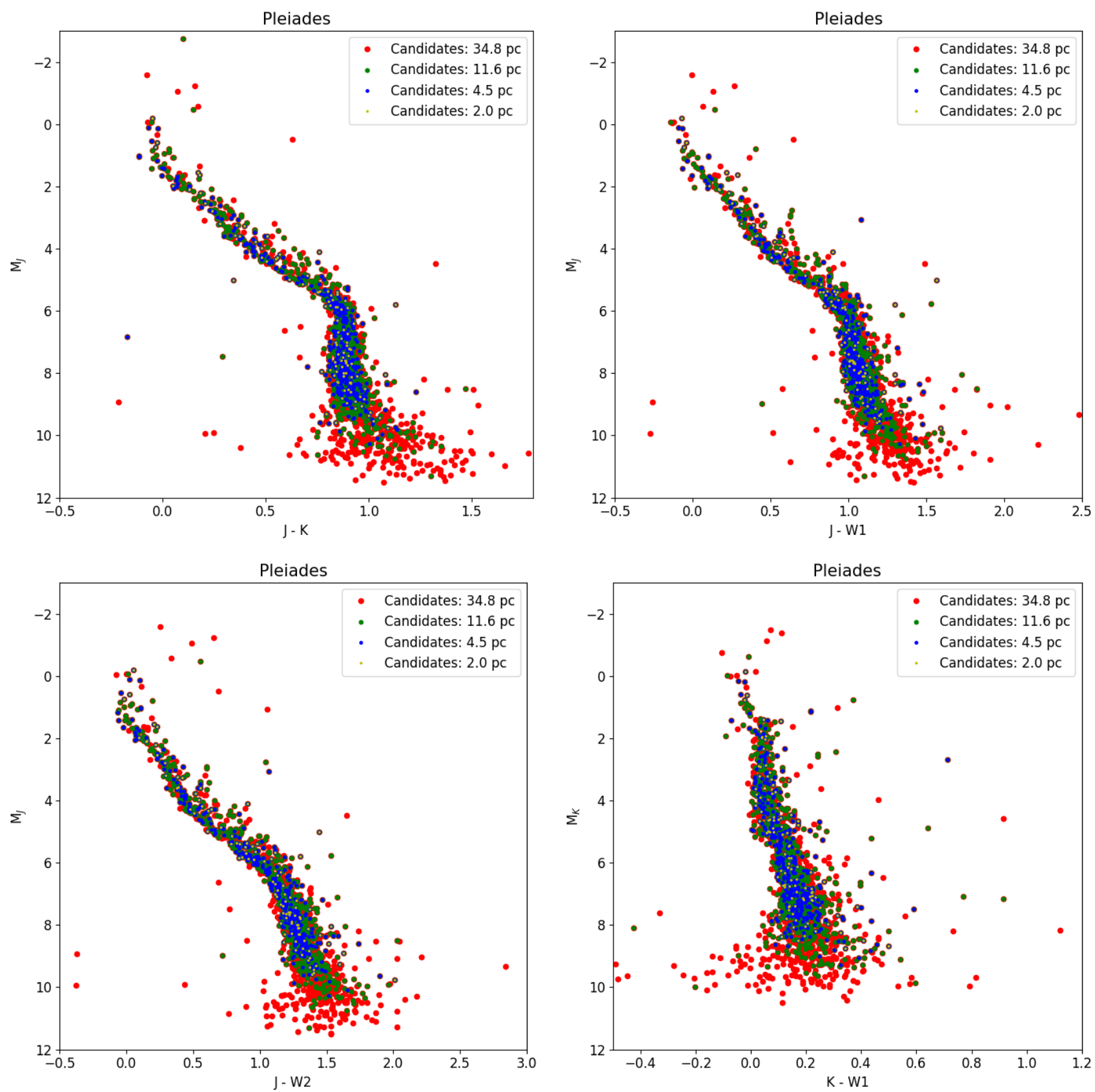

Fig. B.4. Colour-magnitude diagrams for the Pleiades with non-Gaia photometric passbands. Symbols are as in Fig. B.2.

Table B.1. Catalogue of Pleiades member candidates located within the tidal radius of the cluster.

\begin{tabular}{|c|c|c|c|c|c|c|c|c|c|c|c|c|c|c|c|c|}
\hline SourceID & RA & Dec & pmRA & pmDec & Plx & $G$ & $b_{x}$ & $b_{y}$ & $b_{z}$ & $v_{x}$ & $v_{y}$ & $v_{z}$ & $d_{\text {centre }}$ & $c$ & Mass & $\mathrm{RV}$ \\
\hline & deg & deg & mas $\mathrm{yr}^{-1}$ & mas $\mathrm{yr}^{-1}$ & mas & mag & $\mathrm{pc}$ & $\mathrm{pc}$ & $\mathrm{pc}$ & $\mathrm{km} \mathrm{s}^{-1}$ & $\mathrm{~km} \mathrm{~s}^{-1}$ & $\mathrm{~km} \mathrm{~s}^{-1}$ & $\mathrm{pc}$ & & $M_{\odot}$ & $\mathrm{km} \mathrm{s}^{-1}$ \\
\hline 88861709418842496 & 40.974970833896 & 22.655743940905 & 15.587 & -33.165 & 6.712 & 18.172 & -112.22 & 54.06 & -81.76 & - & - & - & 18.615 & 37.934 & 0.114 & - \\
\hline 11268 & 1.714674914238 & 2.655860086477 & 22.806 & 54.808 & 8.078 & 20.067 & -94.15 & 43.94 & -67.30 & - & - & - & 17.527 & 32.715 & 0.059 & - \\
\hline 114833750319750912 & 42.242873946349 & 27.033836378275 & 20.579 & -32.107 & 8.040 & 18.397 & -96.91 & 49.63 & -60.14 & - & - & - & 21.623 & 31.687 & 0.1 & - \\
\hline 128604205745014656 & 42.822225147935 & 28.811112669751 & 41.455 & -65.346 & 7.391 & 20.044 & -106.71 & 55.83 & -61.68 & - & - & - & 8.794 & 30.946 & 0.059 & - \\
\hline 1303 & 42.928644668868 & 31.943391145743 & 30.722 & -44.277 & 7.494 & 20.243 & -106.07 & 59.40 & -55.02 & - & - & - & 5.057 & 33.595 & 0.056 & - \\
\hline 114535958762185600 & 43.052790394089 & 26.245931711561 & 36.093 & -99.403 & 6.614 & 20.782 & -118.65 & 57.73 & -73.83 & - & - & - & 7.584 & 34.715 & 0.049 & - \\
\hline 154 & 6447 & 2669 & 10.41 & -49.344 & 7.199 & 15.072 & -134.90 & 14.18 & -29.92 & - & - & - & 1.687 & & 0.427 & - \\
\hline 3413 & 71.3382241 & 22.763339 & 10.233 & 61 & 7.418 & 20.540 & -130.28 & 6.23 & -34.06 & - & - & - & 2.589 & 32.161 & 0.052 & - \\
\hline 1465 & 71.3718765 & 23.5462027 & 14.408 & -48.123 & 8.025 & 18.367 & -120.63 & 7.06 & -30.40 & - & - & - & 1.414 & 32.545 & 0.102 & - \\
\hline 3413153850924754816 & 71.392270839100 & 23.005022102866 & 7.543 & -57.013 & 6.847 & 20.868 & -141.25 & 7.16 & -36.43 & - & - & - & 3.988 & 35.245 & 0.049 & - \\
\hline 14746 & 71.758353684925 & 25.557751301650 & 17.011 & -41.693 & 7.023 & 15.203 & -138.50 & 11.49 & -31.05 & - & - & - & 13.155 & 34.460 & 0.411 & - \\
\hline 3413681994462425216 & 72.525370378847 & 24.013526031333 & 8.657 & -68.246 & 7.207 & 19.598 & -135.01 & 7.21 & -31.22 & - & - & - & 14.661 & 35.096 & 0.066 & - \\
\hline
\end{tabular}

Notes. We list only a subsample of member candidates. The full table is available at the CDS. 


\section{Appendix C: Tables and diagrams for Praesepe}

We make public via CDS/Vizier the full table of Praesepe candidate members after applying the kinematic analysis described in Sect. 4. The full catalogue contains 1847 sources located within three times the tidal radius with Gaia DR2 data and photometry from several large-scale surveys. Below we show a subset with a limited numbers of columns for space reasons, including source identifiers, coordinates, proper motions, parallaxes, and $G$ magnitudes.
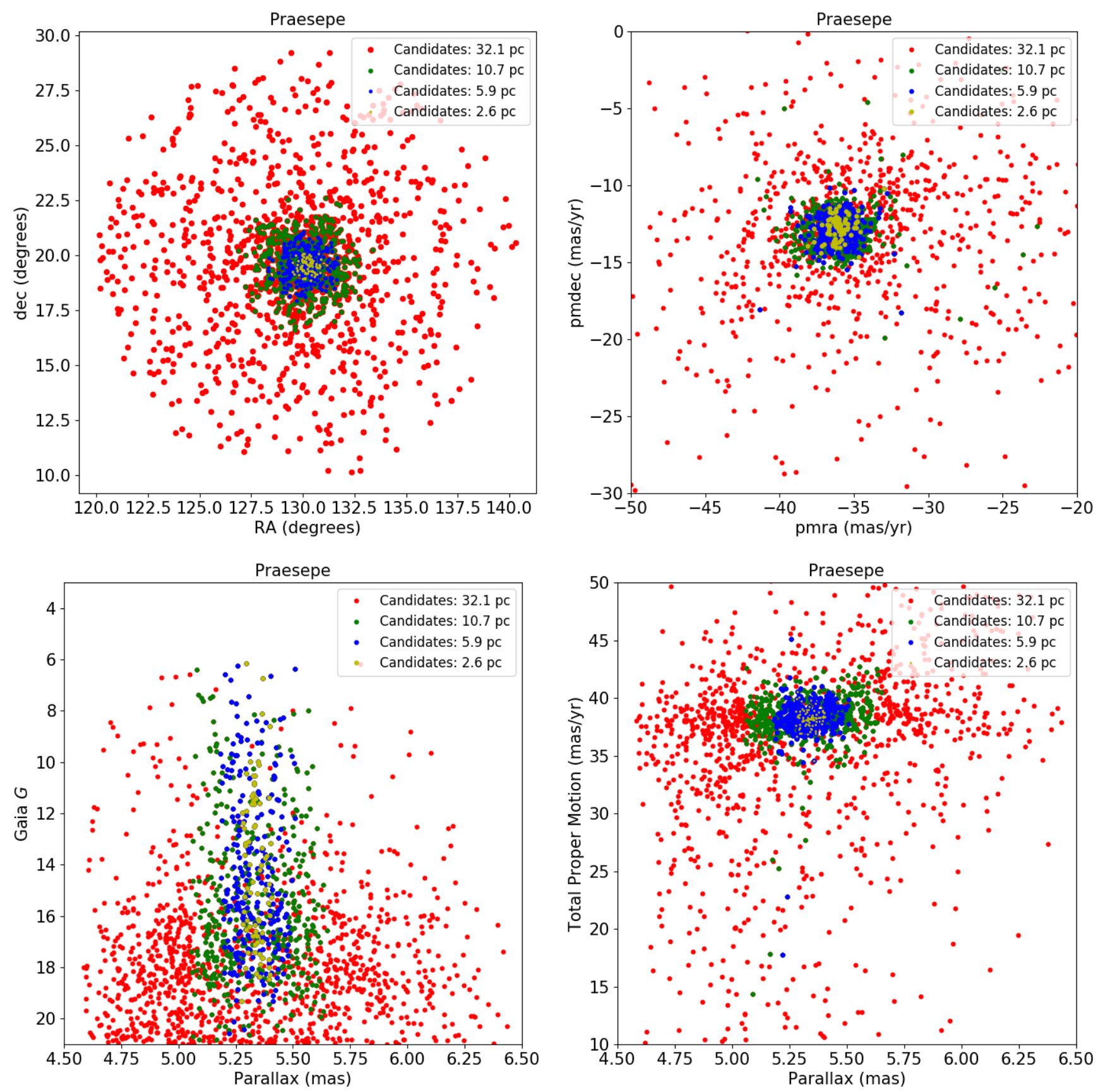

Fig. C.1. Top left: (RA,Dec) diagram showing the position of Praesepe member candidates. Samples at different distances from the cluster centre are highlighted with distinct colours and sizes. Top right: vector point diagram. Bottom left: Gaia magnitude vs. parallax. Bottom right:: total proper motion as a function of parallax. 

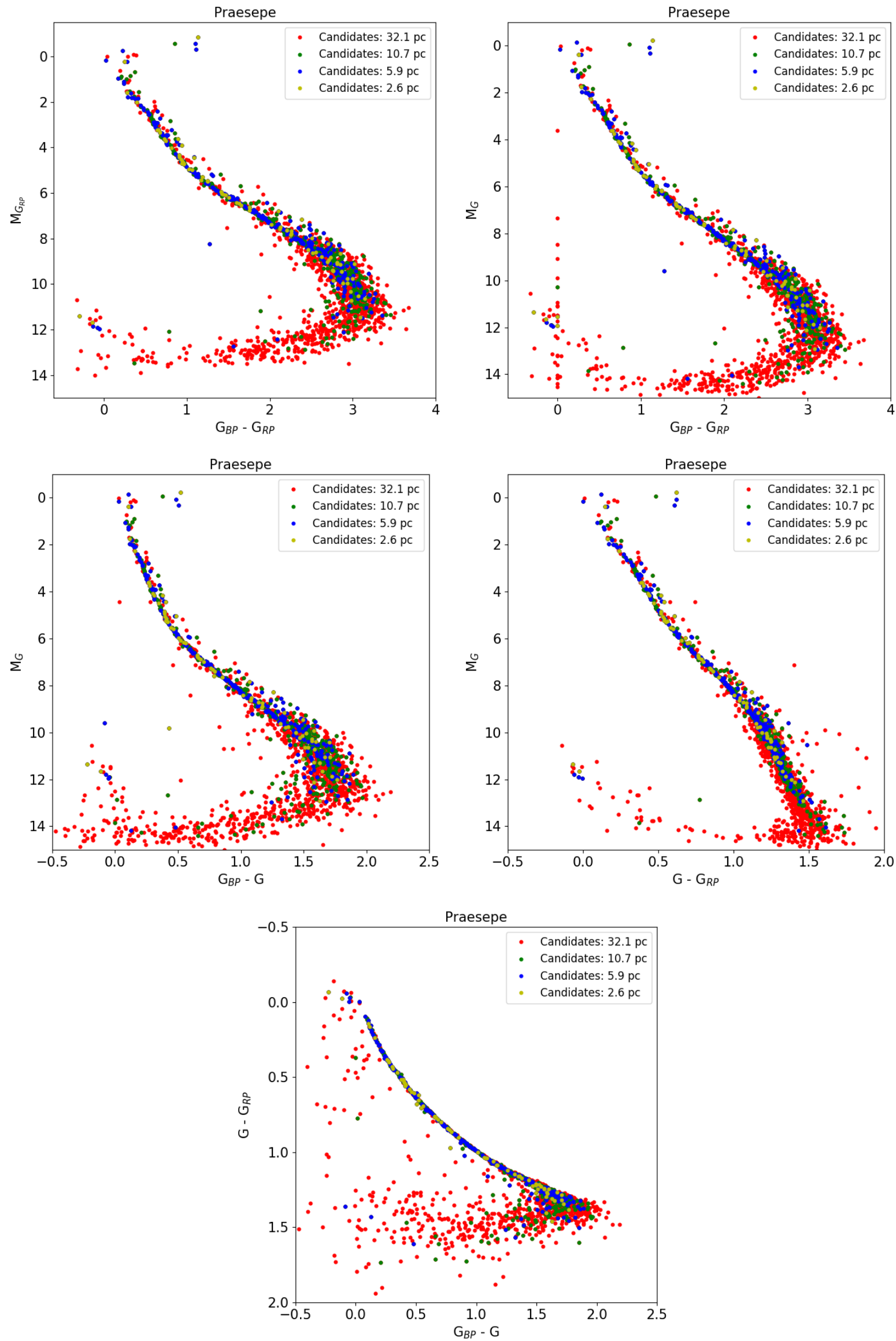

Fig. C.2. Colour-magnitude (top and middle rows) and colour-colour (bottom panel) diagrams with Gaia photometry only for all Praesepe candidates within a radius of $32^{\circ}$ from the cluster centre. We added the full Gaia catalogue in a large region around Praesepe (small grey dots). 
N. Lodieu et al.: A 5D view of the $\alpha$ Per, Pleiades, and Praesepe clusters
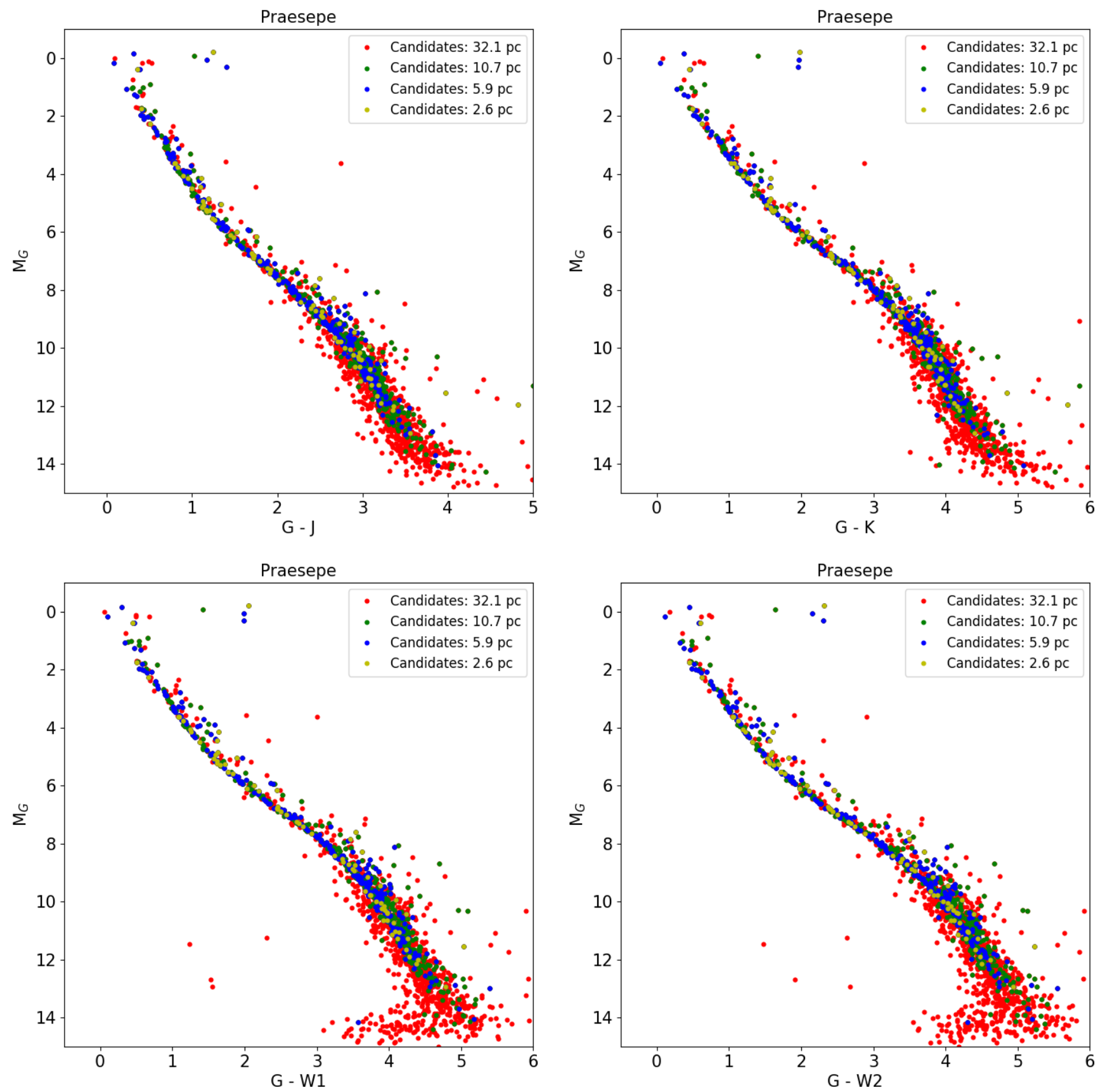

Fig. C.3. Colour-magnitude diagrams for Praesepe combining the Gaia magnitude with infrared photometry from $2 \mathrm{MASS}\left(J+K_{s}\right)$ and AllWISE $(W 1+W 2)$. Symbols are as in Fig. C.2. 

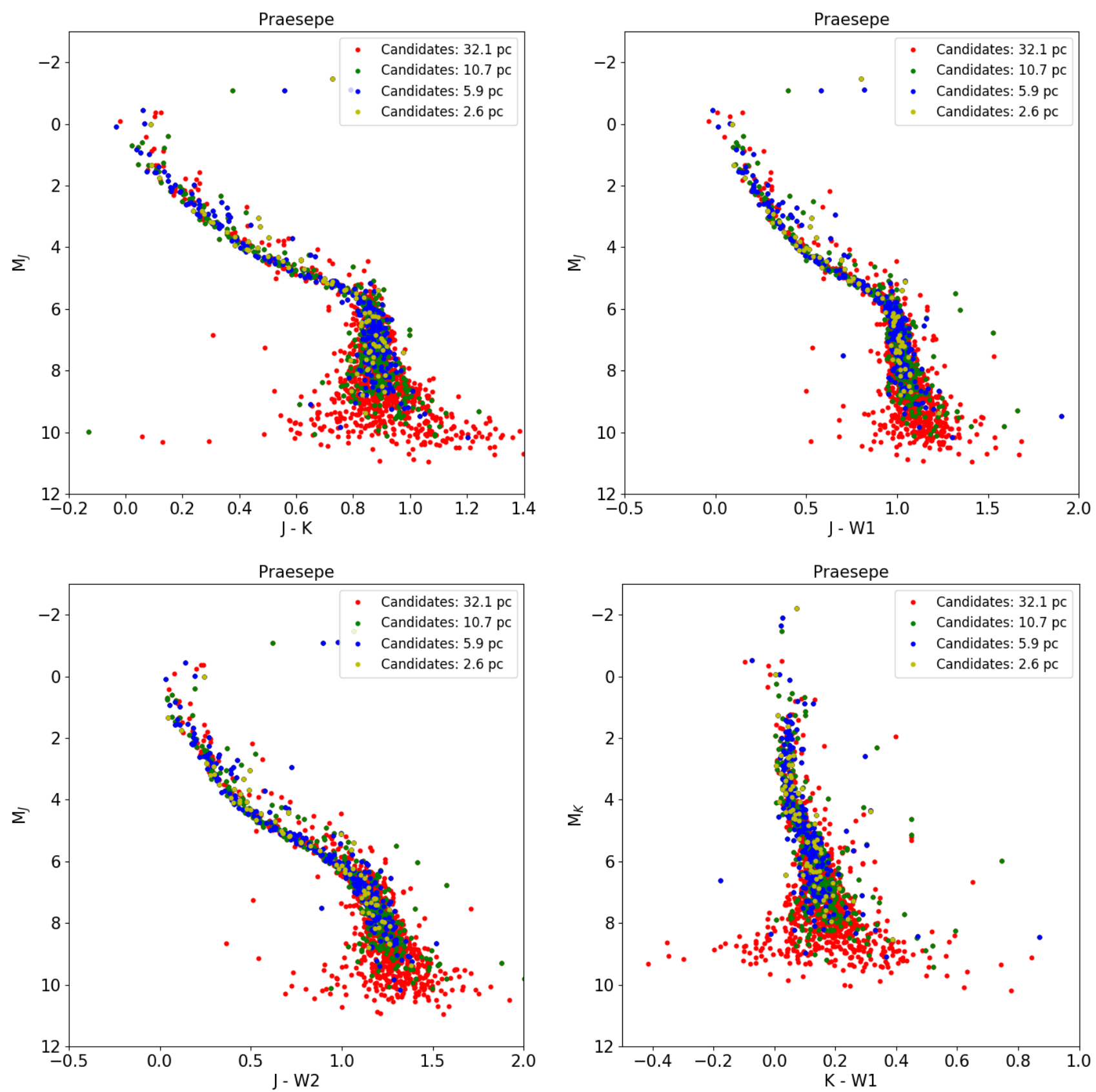

Fig. C.4. Colour-magnitude diagrams for Praesepe with non-Gaia photometric passbands. Symbols are as in Fig. C.2.

Table C.1. Catalogue of Praesepe member candidates located within the tidal radius of the cluster.

\begin{tabular}{|c|c|c|c|c|c|c|c|c|c|c|c|c|c|c|c|c|}
\hline SourceID & RA & Dec & pmRA & pmDec & Plx & $G$ & $b_{x}$ & $b_{y}$ & $b_{z}$ & $v_{x}$ & $v_{y}$ & $v_{z}$ & $d_{\text {centre }}$ & $c$ & Mass & RV \\
\hline & deg & deg & mas $\mathrm{yr}^{-1}$ & mas $\mathrm{yr}^{-1}$ & mas & mag & $\mathrm{pc}$ & $\mathrm{pc}$ & $\mathrm{pc}$ & $\mathrm{km} \mathrm{s}^{-1}$ & $\mathrm{~km} \mathrm{~s}^{-1}$ & $\mathrm{~km} \mathrm{~s}^{-1}$ & $\mathrm{pc}$ & & $M_{\odot}$ & $\mathrm{km} \mathrm{s}^{-1}$ \\
\hline 673332163412736640 & 118.671345250596 & 19.966185887724 & -27.373 & -22.881 & 5.714 & 19.558 & -150.64 & -58.56 & 67.14 & - & - & - & 4.959 & 36.351 & 0.13 & - \\
\hline 668501115479895808 & 118.849980234877 & 18.734957249821 & -22.459 & -13.183 & 5.645 & 17.424 & -151.43 & -62.86 & 67.07 & - & - & - & 18.919 & 35.568 & 0.244 & - \\
\hline 670463498920784512 & 119.311702025846 & 20.475050801747 & -19.259 & -17.779 & 5.829 & 18.536 & -147.12 & -56.39 & 67.87 & - & - & - & 19.606 & 35.713 & 0.172 & - \\
\hline 666720039788305920 & 119.420635803765 & 15.684454972027 & -12.571 & -13.871 & 5.474 & 20.162 & -152.96 & -73.94 & 67.14 & - & - & - & 11.687 & 35.628 & 0.106 & - \\
\hline 670414330135404416 & 119.431265206749 & 20.070160223743 & -19.887 & -9.106 & 5.233 & 20.119 & -163.43 & -64.13 & 75.46 & - & - & - & 11.391 & 33.594 & "" & - \\
\hline 667645008236564864 & 119.448531778971 & 17.921918325649 & -5.426 & -6.718 & 5.219 & 20.816 & -162.27 & -70.86 & 73.21 & - & - & - & 9.975 & 34.246 & "" & - \\
\hline$\ldots$ & & & $\ldots$ & & ... & & & . & ... & $\ldots$ & .. & .. & $\ldots$ & & & \\
\hline 637577896412129024 & 140.294307219569 & 20.557663044063 & -84.731 & -33.850 & 5.501 & 19.828 & -118.67 & -65.39 & 121.19 & - & - & - & 15.877 & 31.236 & - & - \\
\hline 632738395977951488 & 140.474509676173 & 19.042042476738 & -47.127 & -10.999 & 5.838 & 12.636 & -110.19 & -65.80 & 113.43 & -41.03 & -19.46 & -9.11 & 15.909 & 34.370 & 0.787 & 27.84 \\
\hline 632329412012224640 & 140.517789036423 & 18.002924454129 & -49.880 & -14.769 & 5.781 & 17.580 & -110.26 & -69.33 & 113.82 & - & - & - & 5.168 & 34.261 & 0.228 & - \\
\hline 637763168412025984 & 140.562206859202 & 21.348163316669 & -51.545 & -26.420 & 7.102 & 19.399 & -92.01 & -48.82 & 94.76 & - & - & - & 20.744 & 54.317 & 0.137 & - \\
\hline 632722869671684096 & 140.706205038359 & 18.944309012550 & -40.915 & -15.227 & 5.998 & 14.759 & -106.72 & -64.29 & 110.79 & - & - & - & 8.066 & 36.963 & 0.563 & - \\
\hline 632454348315678080 & 141.410495232392 & 18.577579180111 & -40.884 & -10.565 & 5.497 & 16.857 & -114.60 & -71.15 & 122.08 & - & - & - & 5.337 & 34.721 & "" & - \\
\hline
\end{tabular}

Notes. We list only a subsample of member candidates. The full table is available at the CDS. 